\title{
The effect of green tea as an adjunct to scaling and root planing in non-surgical periodontitis therapy: a systematic review
}

\author{
Gartenmann, S J ; Weydlich, Y V ; Steppacher, S L ; Heumann, C ; Attin, T ; Schmidlin, Patrick R
}

\begin{abstract}
OBJECTIVE To provide a systematic overview on the efficacy of green tea catechin as an adjunct to scaling and root planing (SRP) in terms of probing pocket depth (PPD). MATERIALS AND METHODS A systematic literature search was performed using electronic databases in PubMed, Scopus, Medline, Cochrane, CINAHL, and Web of Science on randomized clinical trials up to January 2017. The research question was posed in accordance with PRISMA guidelines. RESULTS The search provided 234 studies. After analyzing the full texts, five studies were included, with four studies qualifying for meta-analysis. Mean PPD reduction was significantly higher ( $\alpha$ $=0.05$ ) when green tea catechin was used as an adjunct to SRP (test group) than with SRP alone (control group). The difference in the reduction was $0.74 \mathrm{~mm}$ [0.35-1.13; 95\% CI]. CONCLUSION The local application of green tea catechin as an adjunct to SRP may result in a beneficial reduction in PPD. Due to the highly heterogeneous data and some risk of bias, however, this data still needs to be interpreted with caution. CLINICAL RELEVANCE The finding suggests that green tea catechin may be a topical adjunct to SRP without negative side effects.
\end{abstract}

DOI: https://doi.org/10.1007/s00784-018-2684-7

Posted at the Zurich Open Repository and Archive, University of Zurich ZORA URL: https://doi.org/10.5167/uzh-167388

Journal Article

Accepted Version

Originally published at:

Gartenmann, S J; Weydlich, Y V; Steppacher, S L; Heumann, C; Attin, T; Schmidlin, Patrick R (2019). The effect of green tea as an adjunct to scaling and root planing in non-surgical periodontitis therapy: a systematic review. Clinical Oral Investigations, 23(1):1-20.

DOI: https://doi.org/10.1007/s00784-018-2684-7 
The effect of green tea as an adjunct to scaling and root planing in nonsurgical periodontitis therapy: a systematic review S.J. Gartenmann ${ }^{1}$, Y.v. Weydlich², S.L. Staub², C. Heumann ${ }^{3}$, T. Attin ${ }^{1}$,
P.R. Schmidlin ${ }^{1}$

${ }^{1}$ Clinic for Preventive Dentistry, Periodontology and Cariology, Center of Dental Medicine, University of Zurich, Zürich, Switzerland

2 Private practices in Switzerland

${ }^{3}$ Department of Statistics, Ludwig-Maximilians-University Munich, Munich, Germany

\section{Corresponding address}

Prof. Dr. Patrick R. Schmidlin

Clinic for Preventive Dentistry, Periodontology and Cariology

Center of Dental Medicine, University of Zurich

Plattenstrasse 11

$\mathrm{CH}-8032$ Zurich

Phone: +41446343417

Fax: $\quad+41446344308$

e-mail: patrick.schmidlin@zzm.uzh.ch

Keywords: Green tea, local application, chronic periodontitis, non-surgical periodontal therapy, local delivery device 


\section{Abstract}

Objective: To provide a systematic overview on the efficacy of green tea catechin as an adjunct to scaling and root planing (SRP) in terms of probing pocket depth (PPD). Materials and Methods: A systematic literature search was performed using electronic databases in PubMed, Scopus, Medline, Cochrane, CINAHL and Web of Science on randomized clinical trials up to January 2017. The research question was posed in accordance with PRISMA guidelines.

Results: The search provided 234 studies. After analyzing the full texts, five studies were included, with four studies qualifying for meta-analysis. Mean PPD reduction was significantly higher $(\alpha=0.05)$ when green tea catechin was used as an adjunct to SRP (test group) than with SRP alone (control group). The difference in the reduction was $0.74 \mathrm{~mm}[0.35-1.13 ; 95 \% \mathrm{Cl}]$.

Conclusion: The local application of green tea catechin as an adjunct to SRP may result in a beneficial reduction in PPD. Due to the highly heterogeneous data and some risk of bias, however, this data still needs to be interpreted with caution.

Clinical Relevance: The finding suggests that green tea catechin may be a topical adjunct to SRP without negative side effects. 


\section{Background}

Periodontitis is an inflammatory disease of the periodontal tissues. It is caused by bacteria as an etiologic factor and modulated by the host [1, 2]. Subgingival populations of bacteria are organized in biofilms. Therefore, the primary goal of periodontal treatment is to eliminate or reduce the pathogenic species on dental surfaces. Scaling and root planing (SRP) still represents the highest standard of care and mechanically disrupts these biofilms. Nevertheless, a complete elimination of the pathogenic biofilm is not always achieved, due in great part to issues of site accessibility $[3,4]$.

In order to overcome the technical problems associated with subgingival biofilm management, previous research has examined numerous local adjunctive antibiotics and antiseptic agents as well as strategies that have been proposed and investigated in order to improve non-surgical therapy. Several local anti-infective and antibiotic agents are adjunctively placed directly into the periodontal pockets and have been shown to have an impact on bacterial biofilm pathogenicity and periodontal healing [5-7]. One such chemotherapeutic agent, chlorhexidine, was integrated into a biodegradable chip and placed in the periodontal pocked [8, 9]. Nevertheless one of the view studies investigating a potential effect on periodontal pockets could not prove a benefit of chlorhexidine chips compared to systemic antibiotics [10]. Another approach in non-surgical periodontal treatment was the local application of antibiotic agents through numerous mechanisms [11]. Yet showing a statistical advantage compare to SRP alone. The use of such agents has decreased in popularity due to manufacturing issues, difficult administration techniques and financial reasons [7]. In addition, the use of locally applied antibiotics requires the use of higher concentrations (bacteriocidal vs. bacteriostatic) due to a rather fast wash-out time in the periodontal pocket. This, in turn, has given rise to growing criticism [12-14].

Hence, there has been increasing interest in natural alternatives. Green tea is one such substance and has gained attention based on promising scientific reports that show its health benefits, especially in beverages [15]. The active ingredients of green tea are polyphenols. Most of them are catechins (flavan-3-ols), which can be categorized in four main groups: Epigallocatechin-3-gallate (EGCG) being the most common type (59\%), followed by epigallocatechin (EGC, 19\%), epicatechin 3 gallate (ECG, 13.6\%) and epicatechin (EC, 6.4\%) [16]. In addition, whole green tea contains 
other antioxidants in the form of vitamins, such as carotinoids (A), ascorbate (C) and tocopherols $(E)[15]$.

Polyphenols act as antioxidants through the induction of antioxidant enzymes such as glutathione S-transferase and super oxid dismutase. Other mechanisms by which catechins have an influence on the oxidation levels are through binding to iron and copper ions, thus reducing the impact of these ions on oxidation reactions. In addition, they prevent the activation of redox-sensitive transcription factors, which are mediators of inflammatory reactions. Catechins can also suppress other oxidation substances, such as nitric oxid synthase, cyclooxygenase 2 (COX-2), lipoxygenase 2 (LOX-2) and xanthine oxidase [15, 17].

Kushiyama and co-workers showed that green tea can have a preventive effect when taken regularly. It may even reduce the progression of an existing periodontitis [18]. Overall, green tea catechins have an anti-oxidant and anti-bacterial effect on pathogens such as Porphyromonas gingivalis and Prevotella intermedia. The mechanism of action is through the inhibiting effect of EGCG and EGC on cysteine proteases of $P$. gingivalis. [19, 20]. In vitro research also suggests an antiinflammatory effect for other inflammatory processes, such as cardiovascular diseases [21].

Although multiple studies have been conducted to find evidence on the therapeutic effects and benefits of green tea, little is known about the use of this substance for periodontally diseased patients. Therefore, this systematic review was undertaken to evaluate the potentially beneficial effects of green tea extract as a topically applied adjunct to SRP during non-surgical periodontal therapy in patients with periodontal disease.

\section{Materials and Methods}

\section{Protocol}

The current systematic review was conducted in accordance with the Preferred Reporting Items of Systematic Reviews (PRISMA) and Meta-analyses statement [22]. The focused question adopted the Population, Intervention, Comparison and Outcomes criteria [23].

The focused question for the present systematic review was: "What is the effect of green tea catechins as an adjunct to scaling and root planing (SRP + GT) compared to SRP and placebo on the PPD in patients with chronic periodontitis? 


\section{Literature search strategy and study selection}

The following electronic search was conducted to identify research articles up to January 2017 dealing with green tea catechin as adjunct to SRP. The databases used for the search were CINAHL, Cochrane Library, MEDLINE, PubMed and Scopus. The following combinations of search terms were used: "periodontal disease" OR "loss" OR "pocket" OR "abscess" OR "abscesses" OR "periodontitis" OR "attachment AND loss" AND "camellia AND chinensis" OR "green tea" OR "epigallocatechin" OR "gallate" OR "gallic acid" (Appendix 1).

Two of the authors (Y.v.W. and S.ST.) independently screened the titles and abstracts for inclusion. The collection of search terms from the electronic database resulted in 234 titles in January 2017. A third reviewer (P.R.S.) re-examined the search. In case of disagreement, the study in doubt was also evaluated by a fourth reviewer (S.E.) and solved through discussion. Finally, eight studies were selected for full text assessment (Fig. 1).

\section{Inclusion and exclusion criteria}

The eligibility criteria for the search included original publications investigating green tea extract as an adjunct to SRP compared to SRP alone. Based on the following criteria, abstracts were included: publications in English, German or Spanish language; randomized clinical trials; extract of green tea explicitly used as an adjunct to non-surgical therapy in patients with chronic periodontitis (SRP); the extract was explicitly used as a local delivery device, either in a strip or gel.

Reasons for excluding publications were as follows: in vitro study, case report, the study population was treated for gingivitis, only green tea and not a green tea extract was used, or green tea was not used in a local delivery device; the study population suffered from a systemic disease (for example diabetes) (Appendix 2).

\section{Data extraction}

From each study, the following data were collected in a data extraction file: number of subjects, chemical composition of the green tea extract, chemical composition of the placebo device, study period, point in time of application of the test and control device, PPD at baseline and study end, PI at baseline and study end and $\mathrm{GI}$ at baseline and study end. 


\section{Data analysis and synthesis}

At baseline and study end, mean and standard deviations were extracted from the studies. A major statistical challenge was the lack of reported standard deviations on the changes in parameters from baseline to endpoint. Additionally, outcomes between different treatments are correlated in a split-mouth design. Therefore, an analysis was conducted between individual values at baseline and endpoint of the two different treatments in the same individual. Probing pocket depths (PPD) were defined as the primary outcome measure, Plaque (PI) and Gingiva Index (GI) as secondary outcome measures. The open source software $R$ with the package "metafor" was used for a random effects analysis according to the method of DerSimonian and Laird [24]. Several combinations for the correlation of the baseline and endpoint values, as well as the correlation between the two measured differences of test and control group reflecting the split-mouth design, were used. The combination with the smallest lower confidence interval found is reported in Table 5 (conservative estimate)

\section{Report of risk of bias for individual studies}

The methodological and reporting quality of the studies included was rated through a modified version of the Cochrane Collaboration's Tool [25] (Appendix 3). The studies of interest, given their adequate reporting of items, were evaluated for quality and risk of bias.

\section{Results}

\section{Study selection}

The electronic data search initially identified 234 studies. After reviewing the titles and abstracts, 226 of these studies were excluded. After analysing the full text of eight studies, four studies had to be excluded; one due to the inclusion criteria of patients being diagnosed with diabetes [26], another due to missing information on study design and sample size [27], the third because no information on study design and sample size was presented. The fourth study got excluded due to the nature of the study with only six participants included into the study [28]. Four studies were designed in a randomized, double-blinded split-mouth model and could be included in the meta-analysis $[29,30,31,32]$. Further, one randomized, double-blinded, 
placebo controlled parallel-group study did not fit the meta-analysis requirements and will be described separately [33]. The Figure 1 demonstrates the selection process in a flow-chart.

\section{Description of characteristics and results}

The characteristics of the study are described in Table 1. Study characteristics, relevant for the specific research question are described as follows:

Population: Two studies included subjects between 30 and 55 years of age [29, 31]. Two studies included patients older than 35 years [32,33]. One study did not provide age [30]. The ethnicity of the subjects was not disclosed. In fact, three studies were performed in India, one in Japan and one in Thailand, which suggests populations of Asian descent. Only one study from Mendoza, Argentina, evaluated a Caucasian population. All studies described chronic periodontitis and a cut-off measurement of $>$ $5 \mathrm{~mm}$ PPD. In some studies, patients had to display > $5 \mathrm{~mm}$ PPD on at least 2 sites bilaterally in order to meet the inclusion criteria. The study conducted by Chava and co-workers included patients with 4 to $6 \mathrm{~mm}$ PPD on $>30 \%$ of sites [31]. All splitmouth designed studies defined the allocation of the PPD measurements in the specific quadrants. The following exclusion criteria were defined by 4 studies: Periodontal treatment with and without antibiotic treatment in the past 6 months, smokers, lactating and pregnant women and systemic diseases. Another study excluded patients who had undergone topical or systemic antibiotic treatment either during the past 60 days or during the study period [30]. The studies included to the meta-analysis examined a total of 34 patients treated with catechin strips and 80 patients treated with catechin gel. However, the number of treated teeth was not declared.

\section{Intervention/Comparison:}

All included studies performed SRP and an adjunct application of either a green tea catechin strip or gel on the test sites. Funosas and co-workers performed either SRP alone or placed a placebo gel as a control. This study evaluated both control group possibilities in different quadrants [30]. Within the identified studies, three publications examined green tea gel and three studies used a hydroxypropyl cellulose strip as carrier for the green tea extract. The local delivery device (LDD) 
was placed once after SRP, except in one studies. Rattanasuwan et al. repeated the application one and two weeks after SRP with no further intervention [33]. Study periods ranged from three weeks to eight months.

\section{Outcomes:}

All studies registered PPD at baseline and study end for the test and control groups. All studies observed sites with at least $5 \mathrm{~mm}$ PPD. In addition to PPD, GI was evaluated by Chave et al., and GI and PL in four other the studies [31].

\section{a) Probing pocket depth (PPD)}

Within the test group, PPD at baseline ranged from 4.93 to $6.43 \mathrm{~mm}$ and at study end from 2.87 to $5.14 \mathrm{~mm}$ (Table 2). Within the control group, PPD at baseline ranged from 4.77 to $5.71 \mathrm{~mm}$ and at study end from 2.83 to $5.14 \mathrm{~mm}$. The reduction of PPD ( $\triangle$ PPD) within the test group ranged from 1.28 to $2.71 \mathrm{~mm}$ with a mean of $1.89 \mathrm{~mm}$ and within the control group from 0.57 to $2.67 \mathrm{~mm}$ with a mean of $1.34 \mathrm{~mm}$. Chava et al. reported a minor standard deviation for $\triangle \mathrm{PPD}$ for the test and control group, 0.07 $\mathrm{mm}$ and $0.02 \mathrm{~mm}$, respectively [31].

\section{b) Gingival index (GI)}

Table 3 displays the baseline GI for the test group, which ranged from 1.67 to 2, and at study end from 0.01 to 0.96 . Equal to the test group, GI at baseline in the control group ranged from 1.67 to 2 and at study end from 0.16 to 1.1. The reduction of $\mathrm{GI}$ within the test group ranged from 0.71 to 1.91 with a mean reduction of 1.21 and within the control group from 0.64 to 1.97 with a mean reduction of 1.1 .

c) Plaque index (PI)

Only two studies documented PI Scores, whereby Hattarki et al. provided identical data for the test and control groups [32, 29]. Mean PL reduction in the test group was 1.12 and in the control group 0.99 (Table 4).

d) Meta-analysis PPD

SRP treatment with adjunctive green tea catechin application resulted in a $0.74 \mathrm{~mm}$ PPD reduction $[0.35,1.13 ; 95 \% \mathrm{Cl}]$ in favour of green tea compared to SRP alone (Table 5). One study presented a very small standard error [31]. To fit statistical 
measurements, the study was removed and the results remained statistically significant with a $0.57 \mathrm{~mm}[0.35,0.78 ; 95 \% \mathrm{Cl}]$ PPD reduction after SRP treatment with green tea application.

e) Rattanasuwan (parallel-group study)

This study was excluded from the meta-analysis because of their parallel-group design and reported no difference between test and control group $(0.04 \mathrm{~mm}$ in favour of SRP + GT) [33]. Nevertheless, the study observed 48 patients over a period of 6 months, where patient populations in the meta-analysis studies ranged from 14 to 50 patients [29, 30,32].

\section{f) Adverse Effects}

Only Chava et al. provided the information that no adverse events occurred during their study. [31]. The other studies did not mention the occurrence of adverse reactions, one way or the other.

\section{g) Bleeding on probing (BOP)}

Only Rattanasuwan et al. evaluated BOP as a secondary outcome, and showed a significantly higher reduction in the test group after three months [33].

\section{Discussion}

This study evaluated the clinical efficacy of topically applied green tea catechin substances as a natural adjunct to SRP, as compared to SRP alone or using a placebo. PPD reduction was the primary outcome parameter for determining efficacy. Five randomized controlled clinical trials with a split-mouth design, and using green tea catechins as an adjunct to SRP, could be included in this systematic review. Overall, the adjunctive application of the test agent resulted in an observed weighed mean difference in PPD reduction of $0.74 \mathrm{~mm}[0.35-1.13 ; 95 \% \mathrm{Cl}$ ] favouring the test group.

Systemic antibiotics as an adjunct to SRP have shown great success in the treatment of periodontal disease, but are associated with the development of bacterial resistance and systemic adverse events [34, 35]. Therefore, the local application of natural alternative substances with a local anti-inflammatory effect, such as green tea catechin, is of great clinical interest. 
The clinical benefit measured appears to be in line with previous reviews on the effect of other antimicrobial substances frequently used as adjuncts in non-surgical periodontal therapy [36, 37, 33]. Only one systematic review evaluated alternative locally applied agents and compared the PPD reduction to SRP alone [36]. The most frequently used and studied antimicrobial agents, chlorhexidine, minocycline and doxycycline, were also investigated in this review. Minocycline, locally applied as a gel or microencapsulated agent, showed a significant PPD reduction, whereas the use of chlorhexidine or doxycycline only displayed a significant effect on CAL gain.

The systematic review by Bonito and co-workers [11] showed an estimated reduction in PPD of $0.49 \mathrm{~mm}$ in favor of locally applied minocycline as an adjunct to SRP compared to SRP alone. Locally applied tetracycline presented a PPD reduction of $0.47 \mathrm{~mm}$. The application of metronidazole and chlorhexidine were below these benchmark results. The review by Matesanz-Pérez [37] showed a PPD reduction value of $0.73 \mathrm{~mm}$ when tetracycline fibers were applied as an adjunct to SRP. Doxycyclin and Minocyclin showed less prominent effects in terms of PPD reduction, with mean values of $0.57 \mathrm{~mm}$ and $0.47 \mathrm{~mm}$. The parallel-group study, which was not included to the meta-analysis treated 48 patients in a 6-month investigation, being the longest study launched. Nevertheless, no benefit in PPD reduction could be observed [33]. The findings of the present systematic review are therefore within the range of earlier findings for well-investigated locally applied antimicrobial or antibiotic agents.

Green tea is a non-fermented product of the Camellia sinensis leaf and has been used historically as a natural medicine for oral diseases [38]. Not surprisingly, all studies included in the current work, except one, were performed in Asia. Because of the applied language restriction, potentially relevant articles, which were not published in English, German or Spanish, were not included in this work. This may expose an overall publication bias. The different concentrations and release patterns of the green tea catechins within the broad range of materials used and the repeated application protocol of the LDD used in at least one of the studies could also lead to biased results and must be taken into consideration when interpreting these positive results.

The studies included and analysed showed a high degree of heterogeneity in terms of population characteristics, different green tea catechins used, carriers, application protocols and study periods. In addition, the sample sizes of the studies included 
were generally rather small. Hirasawa and co-workers only included six patients and therefore got excluded from the systematic review [28]. Three studies included patients ranged from 30 to 55 years of age [29, 31]. One study did not mention the age of the patients included [30]. Furthermore, two studies did not exclude smokers from the study [30, 28]. The inconsistencies of these studies (age, diagnosis and smoking) are potential confounders and could lead to a misinterpretation of the real effect of green tea catechin.

Two studies included teeth with a PPD of $4 \mathrm{~mm}$ to $8 \mathrm{~mm}$ [29, 31], whereas two studies included teeth with a PPD greater than $5 \mathrm{~mm}$ only $[30,32]$. No study classified the general condition of the teeth studied in terms of, i.e. number of roots, furcation involvement or restorations. These anatomical and restorative factors potentially affect the healing response and effects after SRP und may interfere in the effectiveness of the green tea catechins. The study periods varied from 3 weeks to 8 months, which may have an impact on the healing effect as endpoint. In addition, applied maintenance protocols were also inconsistently reported. Unfortunately, due to the small number of included studies, no stratification of the studies was possible. In the current review, two different carriers were used for the sustained release of the green tea catechins. Two studies used a hydroxypropyl cellulose strip [32, 29], while the remaining studies used a carboxymethyl-cellulose-gel, a gel manufactured with carbomer and poloxamer or a thermosensitive hydro-alcoholic-gel. Furthermore, every study used custom-made green tea catechin products, which were manufactured by the research group itself or were purchased from different manufacturers (Sunphenon® or Geltec, both Mumbai, India).

Most of the studies investigated the adjunct of green tea catechin to SRP and compared the results to a control group which received a local placebo device as an adjunct to SRP. In two studies, the control group received only SRP without any local placebo material $[32,29]$. Furthermore, all studies applied the local delivery devices once immediately after SRP.

Although green tea and its base catechins represent a natural product, adverse effects should also be considered. Unfortunately, only one study by Chava and coworkers explicitly reported no adverse effects and assessed this aspect [31].

\section{Conclusion}


The current meta-analysis found that the adjunctive application of green tea catechins result in a beneficial reduction of the probing pocket depth as compared to scaling and root planing with or without placebo. However, due to the high heterogeneity of the data and several risks of bias, this evidence needs to be interpreted with caution. To evaluate the true benefit of green tea catechin in the treatment of chronic periodontitis, further long-term investigations need to be conducted.

Acknowledgements The authors would like to acknowledge the work of Dr. Y. v. Weydlich for his support in the analysis of the data as part of his master thesis.

\section{Compliance with Ethical Standards}

Conflict of interest The authors declare that they have no conflict of interest.

Funding This study was funded by the authors only.

Ethical Approval Ethical approval does not apply to a systematic review Informed Consent Informed consent does not apply to a systematic review 


\section{References}

1. Page RC, Offenbacher S, Schroeder HE, Seymour GJ, Kornman KS. Advances in the pathogenesis of periodontitis: summary of developments, clinical implications and future directions. Periodontology 2000. 1997;14:216-48.

2. Ebersole JL, Dawson D, 3rd, Emecen-Huja P, Nagarajan R, Howard K, Grady ME et al. The periodontal war: microbes and immunity. Periodontology 2000. 2017;75(1):52-115. doi:10.1111/prd.12222.

3. Mombelli A, Schmid B, Rutar A, Lang NP. Persistence patterns of Porphyromonas gingivalis, Prevotella intermedia/nigrescens, and Actinobacillus actinomyetemcomitans after mechanical therapy of periodontal disease. Journal of periodontology. 2000;71(1):14-21. doi:10.1902/jop.2000.71.1.14.

4. Mailoa J, Lin GH, Khoshkam V, MacEachern M, Chan HL, Wang HL. Long-Term Effect of Four Surgical Periodontal Therapies and One Non-Surgical Therapy: A Systematic Review and MetaAnalysis. Journal of periodontology. 2015;86(10):1150-8. doi:10.1902/jop.2015.150159.

5. Tonetti MS, Pini-Prato G, Cortellini P. Principles and clinical applications of periodontal controlled drug delivery with tetracycline fibers. The International journal of periodontics \& restorative dentistry. 1994;14(5):421-35.

6. Haffajee AD, Torresyap G, Socransky SS. Clinical changes following four different periodontal therapies for the treatment of chronic periodontitis: 1-year results. Journal of clinical periodontology. 2007;34(3):243-53. doi:10.1111/j.1600-051X.2006.01040.x.

7. Mombelli A, Cionca N, Almaghlouth A. Does adjunctive antimicrobial therapy reduce the perceived need for periodontal surgery? Periodontology 2000. 2011;55(1):205-16. doi:10.1111/j.16000757.2010.00356.x.

8. Duarte Fde F, Lotufo RF, Pannuti CM. Local delivery of chlorhexidine gluconate in patients with aggressive periodontitis. Journal of the International Academy of Periodontology. 2008;10(1):31-5.

9. Heasman PA, Heasman L, Stacey F, McCracken GI. Local delivery of chlorhexidine gluconate (PerioChip) in periodontal maintenance patients. Journal of clinical periodontology. 2001;28(1):90-5.

10. Kaner D, Bernimoulin JP, Hopfenmuller W, Kleber BM, Friedmann A. Controlled-delivery chlorhexidine chip versus amoxicillin/metronidazole as adjunctive antimicrobial therapy for generalized aggressive periodontitis: a randomized controlled clinical trial. Journal of clinical periodontology. 2007;34(10):880-91. doi:10.1111/j.1600-051X.2007.01122.x.

11. Bonito AJ, Lux L, Lohr KN. Impact of local adjuncts to scaling and root planing in periodontal disease therapy: a systematic review. Journal of periodontology. 2005;76(8):1227-36. doi:10.1902/jop.2005.76.8.1227.

12. Goodson JM, Holborow D, Dunn RL, Hogan P, Dunham S. Monolithic tetracycline-containing fibers for controlled delivery to periodontal pockets. Journal of periodontology. 1983;54(10):575-9. doi:10.1902/jop.1983.54.10.575.

13. Mombelli A, Lehmann B, Tonetti M, Lang NP. Clinical response to local delivery of tetracycline in relation to overall and local periodontal conditions. Journal of clinical periodontology. 1997;24(7):4707.

14. Oosterwaal PJ, Mikx FH, Renggli HH. Clearance of a topically applied fluorescein gel from periodontal pockets. Journal of clinical periodontology. 1990;17(9):613-5.

15. Narotzki B, Reznick AZ, Aizenbud D, Levy Y. Green tea: a promising natural product in oral health. Archives of oral biology. 2012;57(5):429-35. doi:10.1016/j.archoralbio.2011.11.017. 
16. McKay DL, Blumberg JB. The role of tea in human health: an update. Journal of the American College of Nutrition. 2002;21(1):1-13.

17. Cabrera C, Artacho R, Gimenez R. Beneficial effects of green tea--a review. Journal of the American College of Nutrition. 2006;25(2):79-99.

18. Kushiyama M, Shimazaki Y, Murakami M, Yamashita Y. Relationship between intake of green tea and periodontal disease. Journal of periodontology. 2009;80(3):372-7. doi:10.1902/jop.2009.080510.

19. Okamoto M, Sugimoto A, Leung KP, Nakayama K, Kamaguchi A, Maeda N. Inhibitory effect of green tea catechins on cysteine proteinases in Porphyromonas gingivalis. Oral microbiology and immunology. 2004;19(2):118-20.

20. Okamoto M, Leung KP, Ansai T, Sugimoto A, Maeda N. Inhibitory effects of green tea catechins on protein tyrosine phosphatase in Prevotella intermedia. Oral microbiology and immunology. 2003;18(3):192-5.

21. Tijburg LB, Mattern T, Folts JD, Weisgerber UM, Katan MB. Tea flavonoids and cardiovascular disease: a review. Critical reviews in food science and nutrition. 1997;37(8):771-85. doi:10.1080/10408399709527802.

22. Moher D, Shamseer L, Clarke M, Ghersi D, Liberati A, Petticrew M et al. Preferred reporting items for systematic review and meta-analysis protocols (PRISMA-P) 2015 statement. Systematic reviews. 2015;4:1. doi:10.1186/2046-4053-4-1.

23. Miller SA, Forrest JL. Enhancing your practice through evidence-based decision making: PICO, learning how to ask good questions. Journal of Evidence Based Dental Practice. 2001;1(2):136-41. doi:https://doi.org/10.1016/S1532-3382(01)70024-3.

24. DerSimonian R LN. Meta-analysis in clinical trials. Controlled Clinical Trials. 1986;7(3):177-88.

25. Graziani F, Figuero E, Herrera D. Systematic review of quality of reporting, outcome measurements and methods to study efficacy of preventive and therapeutic approaches to periimplant diseases. Journal of clinical periodontology. 2012;39 Suppl 12:224-44. doi:10.1111/j.1600051X.2011.01832.x.

26. Gadagi JS, Chava VK, Reddy VR. Green tea extract as a local drug therapy on periodontitis patients with diabetes mellitus: A randomized case-control study. Journal of Indian Society of Periodontology. 2013;17(2):198-203. doi:10.4103/0972-124X.113069.

27. Yuvaraja M, Reddy NR, Kumar PM, Ravi KS, Alqahtani N. Thermoreversible gel for intrapocket delivery of green tea catechin as a local drug delivery system: An original research. Journal of advanced pharmaceutical technology \& research. 2016;7(4):139-43. doi:10.4103/2231-4040.191422.

28. Hirasawa M, Takada K, Makimura M, Otake S. Improvement of periodontal status by green tea catechin using a local delivery system: a clinical pilot study. Journal of periodontal research. 2002;37(6):433-8.

29. Kudva P, Tabasum ST, Shekhawat NK. Effect of green tea catechin, a local drug delivery system as an adjunct to scaling and root planing in chronic periodontitis patients: A clinicomicrobiological study. Journal of Indian Society of Periodontology. 2011;15(1):39-45. doi:10.4103/0972-124X.82269.

30. Funosas E MA, Pignolo M, Maestri L, Aromando RF, Scozzarro SM, Escovich L, Hermida PS. Efficacy of green tea in the treatment of chronic periodontitis. Avances en Odontoestomatologia. 2005;21(3):159-66.

31. Chava VK, Vedula BD. Thermo-reversible green tea catechin gel for local application in chronic periodontitis: a 4-week clinical trial. Journal of periodontology. 2013;84(9):1290-6. doi:10.1902/jop.2012.120425. 
32. Hattarki SA, Pushpa SP, Bhat K. Evaluation of the efficacy of green tea catechins as an adjunct to scaling and root planing in the management of chronic periodontitis using PCR analysis: A clinical and microbiological study. Journal of Indian Society of Periodontology. 2013;17(2):204-9. doi:10.4103/0972-124X.113071.

33. Rattanasuwan K, Rassameemasmaung S, Sangalungkarn V, Komoltri C. Clinical effect of locally delivered gel containing green tea extract as an adjunct to non-surgical periodontal treatment. Odontology. 2016;104(1):89-97. doi:10.1007/s10266-014-0190-1.

34. Mombelli A, Almaghlouth A, Cionca N, Courvoisier DS, Giannopoulou C. Differential benefits of amoxicillin-metronidazole in different phases of periodontal therapy in a randomized controlled crossover clinical trial. Journal of periodontology. 2015;86(3):367-75. doi:10.1902/jop.2014.140478.

35. Herrera D, van Winkelhoff AJ, Dellemijn-Kippuw N, Winkel EG, Sanz M. Beta-lactamase producing bacteria in the subgingival microflora of adult patients with periodontitis. A comparison between Spain and The Netherlands. Journal of clinical periodontology. 2000;27(7):520-5.

36. Hanes PJ, Purvis JP. Local anti-infective therapy: pharmacological agents. A systematic review. Annals of periodontology. 2003;8(1):79-98. doi:10.1902/annals.2003.8.1.79.

37. Matesanz-Perez P, Garcia-Gargallo M, Figuero E, Bascones-Martinez A, Sanz M, Herrera D. A systematic review on the effects of local antimicrobials as adjuncts to subgingival debridement, compared with subgingival debridement alone, in the treatment of chronic periodontitis. Journal of clinical periodontology. 2013;40(3):227-41. doi:10.1111/jcpe.12026.

38. Radafshar G, Ghotbizadeh M, Saadat F, Mirfarhadi N. Effects of green tea (Camellia sinensis) mouthwash containing $1 \%$ tannin on dental plaque and chronic gingivitis: a double-blinded, randomized, controlled trial. Journal of investigative and clinical dentistry. 2017;8(1). doi:10.1111/jicd.12184. 
Fig. 1: Literature screening procedure (flow-chart)

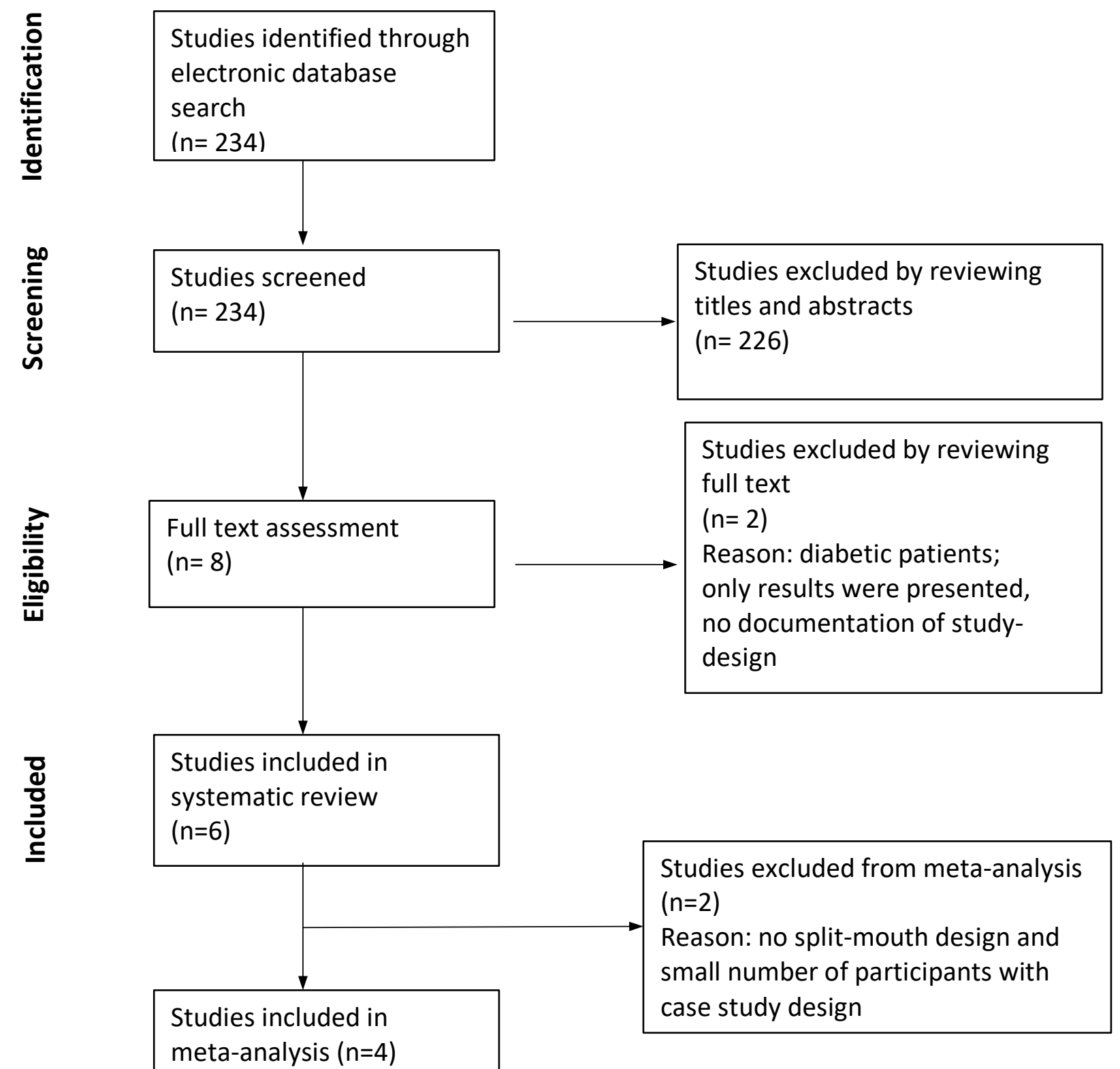


Table 1: Study characteristics of included studies to the review. Last study was excluded from the meta-analysis

\begin{tabular}{|c|c|c|c|c|c|}
\hline $\begin{array}{l}\text { First author } \\
\text { (year of } \\
\text { publication) }\end{array}$ & $\begin{array}{l}\text { Population } \\
\text { characteristics (ethnicity, age, } \\
\text { inclusion criteria) }\end{array}$ & $\begin{array}{l}\text { Number } \\
\text { of } \\
\text { subjects }\end{array}$ & $\begin{array}{l}\text { study design, } \\
\text { intervention }\end{array}$ & $\begin{array}{l}\text { Local delivery device characteristics, point in } \\
\text { time of application, study period }\end{array}$ & Analyzed parameters \\
\hline $\begin{array}{l}\text { Funosas ER } \\
(2005)\end{array}$ & $\begin{array}{l}\text { ethnicity: } \mathrm{nr} \\
\text { age: } \mathrm{nr} \\
\text { inclusion criteria: } \\
\text { chronic periodontitis } \\
\text { at least } 3 \text { sites with } \mathrm{PPD} \geq 5 \mathrm{~mm} \\
\text { and not more than } 2 \mathrm{~mm} C A L \text { in } \\
\text { each quadrant } \\
\text { exclusion criteria: } \\
\text { topical or systemic antibiotic } \\
\text { treatment in the last } 60 \text { days or } \\
\text { during study period }\end{array}$ & 50 & $\begin{array}{l}\text { study design: split mouth } \\
\text { at least three sites in each } \\
\text { quadrant } \\
\text { first quadrant: scaling and } \\
\text { root planing } \\
\text { second quadrant: green tee } \\
\text { gel only } \\
\text { third quadrant: scaling and } \\
\text { root planing \& green tea gel } \\
\text { fourth quadrant: scaling and } \\
\text { root planing \& placebo gel }\end{array}$ & $\begin{array}{l}\text { test gel: } \\
\text { carboximetyl cellulose } 1.5 \mathrm{~g}, \\
\text { green tea extract } 1 \mathrm{~g} \text {, distilled water } 100 \mathrm{ml} \text { in a } \\
1 \% \text { solution } \\
\text { placebo gel: } \\
\text { carboximethyl cellulose } 1.5 \mathrm{~g}, \\
\text { distilled water } 100 \mathrm{ml} \\
\text { point in time of application: } \\
\text { once, at baseline after scaling and root planing } \\
\text { study period: } 30 \text { days }\end{array}$ & $\begin{array}{l}\text { probing pocket depth } \\
\text { plaque index (Silness and Loe 1967) } \\
\text { gingiva Index (Loe and Silness 1963) } \\
\text { bleeding on probing (Van der Velden, 1979) } \\
\text { microbiological sample assessed for: } \\
\text { Prevotella species } \\
\text { Porphyromonas species } \\
\text { Bacteropodes species } \\
\text { Propionibacterium species } \\
\text { Actinomyces species }\end{array}$ \\
\hline Kudva (2011) & $\begin{array}{l}\text { ethnicity: } \mathrm{nr} \\
30 \text { to } 55 \text { years } \\
\text { inclusion criteria: } \\
\text { chronic periodontitis } \\
\text { at least } 2 \text { pockets of } 5-8 \mathrm{~mm} \text { in } \\
\text { contralateral quadrants } \\
\text { exclusion criteria: } \\
\text { non-surgical or surgical periodontal } \\
\text { therapy in the last } 6 \text { month } \\
\text { topical or systemic antibiotic } \\
\text { treatment in the last } 6 \text { month } \\
\text { systemic diseases } \\
\text { pregnant or lactating mothers } \\
\text { smokers } 20 \text { scoreable teeth } \\
\text { less than } 20\end{array}$ & 14 & $\begin{array}{l}\text { study design: split mouth } \\
\text { at least two sites in the } \\
\text { contralateral quadrants } \\
\text { test sites: } \\
\text { scaling and root planing \& } \\
\text { green tea catechin strip } \\
\text { (HPC) } \\
\text { control sites: } \\
\text { scaling and root planing } \\
\text { alone }\end{array}$ & $\begin{array}{l}\text { test strip: } \\
\text { catechins used from green tea powder and } \\
\text { hydroxypropyl cellulose (HPC) as the carrier } \\
\text { point in time of application: } \\
\text { once, at baseline after scaling and root planing } \\
\text { study period: } 21 \text { days }\end{array}$ & $\begin{array}{l}\text { probing pocket depth } \\
\text { plaque index (Silness and Loe 1967) } \\
\text { gingiva Index (Loe and Silness 1963) } \\
\text { microbiological sample assessed for: } \\
\text { Porphyromonas gingivalis } \\
\text { Prevotella intermedia } \\
\text { Aggregatibacter actinomycemcomitans } \\
\text { Fuisbacterium species } \\
\text { Capnocytophaga species }\end{array}$ \\
\hline
\end{tabular}




\begin{tabular}{|c|c|c|c|c|c|}
\hline $\begin{array}{l}\text { First author } \\
\text { (year of } \\
\text { publication) }\end{array}$ & $\begin{array}{l}\text { Population } \\
\text { characteristics (ethnicity, age, } \\
\text { inclusion criteria) }\end{array}$ & $\begin{array}{l}\text { Number } \\
\text { of } \\
\text { subjects }\end{array}$ & study design, intervention & $\begin{array}{l}\text { Local delivery device characteristics, point in } \\
\text { time of application, study period }\end{array}$ & Analyzed parameters \\
\hline $\begin{array}{l}\text { Chava VK } \\
\text { (2013) }\end{array}$ & $\begin{array}{l}\text { ethnicity: } \mathrm{nr} \\
28 \text { to } 40 \text { years } \\
\text { inclusion criteria: } 1 \text { ) chronic } \\
\text { periodontitis based on local } \\
\text { factors, associated clinical findings } \\
\text { with probing depths of } 4 \text { to } 6 \mathrm{~mm} \text { in } \\
>30 \% \text { of sites 2) patients willing to } \\
\text { participate in the study and } \\
\text { maintain regular appointments } \\
\text { exclusion critera: } 1 \text { ) systemic } \\
\text { diseases 2) topical or antimicrobial } \\
\text { treatment in the last } 6 \text { months, } \\
\text { including the use of mouthwash } 3 \text { ) } \\
\text { periodontal treatment in the past } 6 \\
\text { months } 4 \text { ) pregnant and lactating } \\
\text { mothers 5) smokers }\end{array}$ & 30 & $\begin{array}{l}\text { study design: } \\
\text { controlled, split-mouth single- } \\
\text { evaluator masked study } \\
\text { two sites with identical } \\
\text { probing depths in the } \\
\text { contralateral quadrants } \\
\text { between } 4 \text { - } 6 \mathrm{~mm} \\
\text { test sites: } \\
\text { phase one therapy (scaling \& } \\
\text { root planing) \& green tea gel } \\
\text { control sites: } \\
\text { phase one therapy (scaling \& } \\
\text { root planing) \& placebo gel }\end{array}$ & $\begin{array}{l}\text { test gel: } \\
\text { to the } 1 \% \text { weight/volume }(1 \mathrm{~g} / 100 \mathrm{~mL}) \text { solution } \\
\text { of green tea extract (Geltec, Mumbai, India) in } \\
\text { distilled water, carbomer (Carbopol } 934 \text {, Loba } \\
\text { Chemie, Mumbai, India) was added in fixed } \\
\text { quantity ( } 1.5 \% \text { weight/volume). Poloxamer } 407 \\
\text { at } 22 \% \text { weight/volume (Pluronic F-127, Sigma- } \\
\text { Aldrich, St. Louis, MO) was added } \\
\text { Total drug content > } 97 \% \text {, (10 mg } / \mathrm{ml}) \\
\text { point in time of application: } \\
\text { once, at baseline after scaling and root planing } \\
\text { study period: } 4 \text { weeks }\end{array}$ & $\begin{array}{l}\text { probing pocket depth } \\
\text { gingiva Index (Loe and Silness 1963) } \\
\text { relative clinical attachment loss } \\
\text { in vitro release pattern of green tea extract gel }\end{array}$ \\
\hline $\begin{array}{l}\text { Hattarki SA } \\
\text { (2013) }\end{array}$ & $\begin{array}{l}\text { ethnicity: } \mathrm{nr} \\
\text { above } 35 \text { years } \\
\text { inclusion criteria: untreated chronic } \\
\text { periodontitis, age }>35 \text { years, } \\
\text { probing depth }>5 \text { mm, more than } \\
20 \text { natural teeth } \\
\text { exclusion criteria: medically } \\
\text { compromised patients, antibiotic or } \\
\text { antimicrobial therapy in the past } 6 \\
\text { months, smokers, pregnant and } \\
\text { lactating mothers }\end{array}$ & $\begin{array}{l}20, \\
40 \text { sites }\end{array}$ & $\begin{array}{l}\text { study design: } \\
\text { randomized placebo } \\
\text { controlled clinical trial, split } \\
\text { mouth design, } 2 \text { sites in the } \\
\text { contralateral quadrants with } \\
\text { pocket probing depths over } 5 \\
\mathrm{~mm} \\
\text { test sites: full mouth scaling } \\
\text { and root planing \& green tea } \\
\text { catechins strip covered by } \\
\text { Coe-pack } \\
\text { control sites: full mouth } \\
\text { scaling and root planning } \\
\text { alone } \\
\end{array}$ & $\begin{array}{l}\text { test strip: } \\
\text { hydroxyproply cellulose containing catechins } \\
\text { (Ambe Phyto Extracts Pvt. Ltd., Hasanpur, Delhi) } \\
\text { point in time of application: } \\
\text { once, at baseline after scaling and root planing } \\
\text { study period: } 5 \text { weeks }\end{array}$ & $\begin{array}{l}\text { probing pocket depth } \\
\text { plaque index (Silness and Loe 1967) } \\
\text { gingiva Index (Loe and Silness 1963) } \\
\text { microbiological analysis of red complex } \\
\text { organism: Treponema denticola, Tannerella } \\
\text { forsythia, Prophyromonas gingivalis }\end{array}$ \\
\hline $\begin{array}{l}\text { Rattanasuwan } \\
\mathrm{K}(2014)\end{array}$ & $\begin{array}{l}\text { ethnicity: } \mathrm{nr} \\
\text { above } 35 \text { years } \\
\text { inclusion criteria: chronic } \\
\text { periodontitis, no systemic } \\
\text { complicating factors, not allergic to } \\
\text { green tea or a product of green } \\
\text { tea, probing pocket depths of } 5 \text { - } \\
10 \text { mm without furcation } \\
\text { involvement, caries or restoration } \\
\text { exclusion criteria: smokers, } \\
\text { pregnant or lactating mothers, } \\
\text { antibiotic therapy in the past } 6 \\
\text { months, received a periodontitis } \\
\text { therapy in the past } 6 \text { months }\end{array}$ & 48 & $\begin{array}{l}\text { study design: } \\
\text { randomized, double-blind, } \\
\text { placebo controlled cohort } \\
\text { study } \\
\text { test sites: } \\
\text { full mouth scaling and root } \\
\text { planning \& green tea gel } \\
\text { application } \\
\text { control sites: } \\
\text { full mouth scaling and root } \\
\text { planning \& placebo gel } \\
\text { application }\end{array}$ & $\begin{array}{l}\text { test gel: } \\
\text { themosensitive gel containing } 12 \% \mathrm{w} / \mathrm{w} \text { of green } \\
\text { tea extract (provided not less than } 80 \% \text { of } \\
\text { catechins) } \\
\text { control gel: } \\
\text { same ingredients as the green tea gel without } \\
\text { green tea extract } \\
\text { point in time of application: } \\
\text { once, at baseline after scaling and root planing } \\
\text { gel application was repeated after } 1 \text { and } 2 \text { weeks } \\
\text { without scaling and root planing } \\
\text { study period: } 6 \text { months after last gel application }\end{array}$ & $\begin{array}{l}\text { probing pocket depth } \\
\text { clinical attachment level } \\
\text { gingiva Index (Loe and Silness 1963) } \\
\text { bleeding on probing (Ainamo and Bay) } \\
\text { full mouth plaque score (O'Leary) } \\
\text { microbiological analysis of red complex } \\
\text { organism: Treponema denticola, Tannerella } \\
\text { forsythia, Prophyromonas gingivalis }\end{array}$ \\
\hline
\end{tabular}


Table 2: Results of probing pocket depth.

\begin{tabular}{|c|c|c|c|c|c|c|c|c|}
\hline $\begin{array}{l}\text { Reference } \\
\text { (year of } \\
\text { publication) }\end{array}$ & \begin{tabular}{|l|} 
PPD $_{\text {Test }}$ \\
Baseline \\
[mm]
\end{tabular} & \begin{tabular}{|l|} 
PPD \\
End [mm]
\end{tabular} & $\begin{array}{l}\text { PPD control } \\
\text { Baseline } \\
\text { [mm] }\end{array}$ & $\begin{array}{l}\text { PPD control } \\
\text { End [mm] }\end{array}$ & \begin{tabular}{|l|} 
Intergroup \\
$\Delta$ PPD $_{\text {Test }}$ \\
{$[\mathrm{mm}]$}
\end{tabular} & \begin{tabular}{|l|} 
Intergroup \\
$\Delta$ \\
PPD \\
{$[\mathrm{mm}]$} \\
\end{tabular} & $\begin{array}{l}\text { Intergroup } \\
\text { p-value }\end{array}$ & $\begin{array}{l}\text { Comparison } \\
\Delta \text { PPD } \text { Test }^{-} \\
\Delta \text { PPD control } \\
{[\mathrm{mm}]}\end{array}$ \\
\hline $\begin{array}{l}\text { Funosas ER } \\
\text { (2005) }\end{array}$ & $5.43 \pm 0.69$ & $3.59 \pm 0.87$ & $5.35 \pm 0.55$ & $4.06 \pm 0.61$ & $1.84 \pm \mathrm{nr}$ & $1.29 \pm \mathrm{nr}$ & $\mathrm{nr}$ & $0.55 \pm n$ \\
\hline Kudva (2011) & $6.43 \pm 0.49$ & $5.14 \pm 0.64$ & $5.71 \pm 0.45$ & $5.14 \pm 0.35$ & $1.28 \pm 0.70$ & $0.57 \pm 0.49$ & $<0.001$ & $0.71 \pm n r$ \\
\hline Chava VK (2013) & $4.93 \pm 0.58$ & $2.87 \pm 0.51$ & $4.77 \pm 0.50$ & $3.80 \pm 0.48$ & $2.06 \pm 0.07$ & $0.97 \pm 0.02$ & $<0.0001$ & $1.09 \pm n r$ \\
\hline $\begin{array}{l}\text { Hattarki SA } \\
(2013)\end{array}$ & $6.20 \pm 1.28$ & $4.60 \pm 1.23$ & $5.65 \pm 0.99$ & $4.45 \pm 1.23$ & $1.60 \pm 0.68$ & $1.20 \pm 0.76$ & 0.089 & $0.40 \pm n r$ \\
\hline $\begin{array}{l}\text { Rattanasuwan K } \\
\text { (2014) }\end{array}$ & $5.84 \pm 1.11$ & $3.13 \pm 1.20$ & $5.50 \pm 0.91$ & $2.83 \pm 1.14$ & $2.71 \pm \mathrm{nr}$ & $2.67 \pm \mathrm{nr}$ & 0.236 & $0.04 \pm n$ \\
\hline Mean & & & & & $1.89 \pm \mathrm{nr}$ & $1.34 \pm \mathrm{nr}$ & & \\
\hline
\end{tabular}


Table 3: Results of gingiva index

\begin{tabular}{|c|c|c|c|c|c|c|c|c|}
\hline $\begin{array}{l}\text { Reference } \\
\text { (year of } \\
\text { publication) }\end{array}$ & $\begin{array}{l}\text { Gl Test } \\
\text { Baseline }\end{array}$ & GITest End & $\begin{array}{l}\text { Glcontrol } \\
\text { Baseline }\end{array}$ & $\begin{array}{l}\text { Glcontrol } \\
\text { End }\end{array}$ & $\begin{array}{l}\text { Intergroup } \\
\Delta \mathrm{G}_{\text {Test }}\end{array}$ & $\begin{array}{l}\text { Intergroup } \\
\Delta \text { Gl }\left.\right|_{\text {control }}\end{array}$ & $\begin{array}{l}\text { Intergrou } \\
\text { p p-value }\end{array}$ & $\begin{array}{l}\text { Comparison } \\
\Delta \text { Gl } \text { Test }^{-} \\
\Delta \text { PPD control }\end{array}$ \\
\hline $\begin{array}{l}\text { Funosas ER } \\
(2005)\end{array}$ & $1.85 \pm 0.33$ & $0.75 \pm 0.04$ & $1.67 \pm 0.41$ & $0.97 \pm 0.05$ & $1.1 \pm \mathrm{nr}$ & $0.7 \pm \mathrm{nr}$ & $\overline{\mathrm{nr}}$ & $0.40 \pm \mathrm{nr}$ \\
\hline Kudva (2011) & $1.67 \pm 0.37$ & $0.96 \pm 0.09$ & $1.75 \pm 0.40$ & $1.11 \pm 0.12$ & $0.71 \pm 0.39$ & $0.64 \pm 0.35$ & $>0.05$ & $0.07 \pm n r$ \\
\hline Chava VK (2013) & $1.92 \pm 0.24$ & $0.01 \pm 0.04$ & $1.95 \pm 0.16$ & $0.16 \pm 0.11$ & $1.91 \pm 0.20$ & $1.97 \pm 0.05$ & $<0.0023$ & $0.12 \pm n r$ \\
\hline $\begin{array}{l}\text { Hattarki SA } \\
(2013)\end{array}$ & $2.00 \pm 0.72$ & $0.85 \pm 0.36$ & $2.00 \pm 0.72$ & $0.90 \pm 0.31$ & $1.15 \pm 0.58$ & $1.1 \pm 0.64$ & $\mathrm{nr}$ & $0.05 \pm \mathrm{nr}$ \\
\hline $\begin{array}{l}\text { Rattanasuwan K } \\
\text { (2014) }\end{array}$ & $1.95 \pm 0.22$ & $0.78 \pm 0.79$ & $2.00 \pm 0.33$ & $0.75 \pm 0.82$ & $1.17 \pm \mathrm{nr}$ & $1.25 \pm \mathrm{nr}$ & $\mathrm{nr}$ & $-0.08 \pm n r$ \\
\hline Mean & & & & & $1.20 \pm \mathrm{nr}$ & $1.13 \pm \mathrm{nr}$ & & \\
\hline
\end{tabular}


Table 4 : Results of plaque index

\begin{tabular}{|c|c|c|c|c|c|c|c|}
\hline $\begin{array}{l}\text { REFERENCE } \\
\text { (YEAR OF } \\
\text { PUBLICATION) }\end{array}$ & $\begin{array}{l}\text { PItEST } \\
\text { BASELINE }\end{array}$ & PItest END & $\begin{array}{l}\text { PICONTROL } \\
\text { BASELINE }\end{array}$ & $\begin{array}{l}\text { PICONTROL } \\
\text { END }\end{array}$ & $\begin{array}{l}\text { INTERGROUP } \\
\Delta \text { PITEST }\end{array}$ & $\begin{array}{l}\text { INTERGROUP } \\
\Delta \text { PICONTROL }\end{array}$ & \begin{tabular}{|l} 
INTERGROUP \\
P-VALUE
\end{tabular} \\
\hline FUNOSAS ER (2005) & $\mathrm{nr}$ & $\overline{\mathrm{nr}}$ & $\overline{\mathrm{nr}}$ & $\mathrm{nr}$ & $\overline{\mathrm{nr}}$ & $\overline{\mathrm{nr}}$ & $\overline{\mathrm{nl}}$ \\
\hline KUDVA (2011) [38] & $2.10 \pm 0.55$ & $0.78 \pm 0.25$ & $2.25 \pm 0.53$ & $1.18 \pm 0.17$ & $1.33 \pm 0.56$ & $1.07 \pm 0.48$ & $>0.05$ \\
\hline CHAVA VK (2013) & $\mathrm{nr}$ & $\mathrm{nr}$ & $\overline{\mathrm{nr}}$ & $\mathrm{nr}$ & $\mathrm{nr}$ & $\mathrm{nr}$ & $\mathrm{nr}$ \\
\hline HATTARKI SA (2013) & $1.80 \pm 0.61$ & $0.90 \pm 0.31$ & $1.80 \pm 0.61$ & $0.90 \pm 0.31$ & $0.90 \pm 0.55$ & $0.90 \pm 0.55$ & $\overline{1}$ \\
\hline $\begin{array}{l}\text { RATTANASUWAN K } \\
\text { (2014) }\end{array}$ & $\mathrm{nr}$ & $\mathrm{nr}$ & $\overline{\mathrm{nr}}$ & $\mathrm{nr}$ & $\mathrm{nr}$ & $\mathrm{nr}$ & $\overline{\mathrm{nl}}$ \\
\hline MEAN & & & & & $1.12 \pm \mathrm{nr}$ & $0.99 \pm \mathrm{nr}$ & \\
\hline
\end{tabular}




\begin{tabular}{|c|c|c|}
\hline Step & Query & Hits \\
\hline 1 & $\begin{array}{l}\text { Search (((periodontal[tiab] OR parodontal[tiab] OR paradontal[tiab]) } \\
\text { AND (disease[tiab] OR diseases[tiab] OR loss[tiab] OR pocket[tiab] OR } \\
\text { pockets[tiab] OR abscess[tiab] OR abscesses[tiab] OR index[tiab])) OR } \\
\text { (pericementitides[tiab] OR pericementitis[tiab] OR periodontitides[tiab] } \\
\text { OR periodontitis[tiab] OR periodontoses[tiab] OR periodontosis[tiab] } \\
\text { OR paradontitis[tiab] OR parodontitis[tiab]) OR (attachment[tiab] AND } \\
\text { loss[tiab]) OR ((clinical[tiab] OR periodontal[tiab] OR parodontal[tiab] } \\
\text { OR paradontal[tiab]) AND attachment[tiab])) }\end{array}$ & 49475 \\
\hline 2 & $\begin{array}{l}\text { Search (((camellia[tiab] AND (chinensis[tiab] OR sinensis[tiab])) OR } \\
\text { ("green tea" [tiab] OR epigallocatechin[tiab] OR gallate[tiab] OR "gallic } \\
\text { acid"[tiab] OR Veregen[tiab] OR Exolise[tiab])) }\end{array}$ & 14095 \\
\hline 3 & $\begin{array}{l}\text { Search (((()eriodontal[tiab] OR parodontal[tiab] OR paradontal[tiab]) } \\
\text { AND (disease[tiab] OR diseases[tiab] OR loss[tiab] OR pocket[tiab] OR } \\
\text { pockets[tiab] OR abscess[tiab] OR abscesses[tiab] OR index[tiab])) OR } \\
\text { (pericementitides[tiab] OR pericementitis[tiab] OR periodontitides[tiab] } \\
\text { OR periodontitis[tiab] OR periodontoses[tiab] OR periodontosis[tiab] } \\
\text { OR paradontitis[tiab] OR parodontitis[tiab]) OR (attachment[tiab] AND } \\
\text { loss[tiab]) OR ((clinical[tiab] OR periodontal[tiab] OR parodontal[tiab] } \\
\text { OR paradontal[tiab]) AND attachment[tiab])))) AND (((((camellia[tiab] } \\
\text { AND (chinensis[tiab] OR sinensis[tiab])) OR ("green tea"[tiab] OR } \\
\text { epigallocatechin[tiab] OR gallate[tiab] OR "gallic acid"[tiab] OR } \\
\text { Veregen[tiab] OR Exolise[tiab]))) }\end{array}$ & 57 \\
\hline 4 & $\begin{array}{l}\text { Search ((inprocess[sb])) OR (publisher[sb] NOT pubstatusnihms NOT } \\
\text { pubstatuspmcsd NOT pmcbook) }\end{array}$ & 1077566 \\
\hline 5 & $\begin{array}{l}\text { Search (((((()eriodontal[tiab] OR parodontal[tiab] OR paradontal[tiab]) } \\
\text { AND (disease[tiab] OR diseases[tiab] OR loss[tiab] OR pocket[tiab] OR } \\
\text { pockets[tiab] OR abscess[tiab] OR abscesses[tiab] OR index[tiab])) OR } \\
\text { (pericementitides[tiab] OR pericementitis[tiab] OR periodontitides[tiab] } \\
\text { OR periodontitis[tiab] OR periodontoses[tiab] OR periodontosis[tiab] } \\
\text { OR paradontitis[tiab] OR parodontitis[tiab]) OR (attachment[tiab] AND } \\
\text { loss[tiab]) OR ((clinical[tiab] OR periodontal[tiab] OR parodontal[tiab] } \\
\text { OR paradontal[tiab]) AND attachment[tiab])))) AND (((((camellia[tiab] } \\
\text { AND (chinensis[tiab] OR sinensis[tiab])) OR ("green tea"[tiab] OR } \\
\text { epigallocatechin[tiab] OR gallate[tiab] OR "gallic acid"[tiab] OR } \\
\text { Veregen[tiab] OR Exolise[tiab]))))) AND (((inprocess[sb])) OR } \\
\text { (publisher[sb] NOT pubstatusnihms NOT pubstatuspmcsd NOT } \\
\text { pmcbook)) }\end{array}$ & 8 \\
\hline
\end{tabular}


5th Joint Meeting of the European Tissue Repair Society and the Wound Healing Society. Wound Repair \& Regeneration. 2009;17(4):A54-871p. Abdulbaqi HR, Himratul-Aznita WH, Baharuddin NA. Anti-plaque effect of a synergistic combination of green tea and Salvadora persica L. against primary colonizers of dental plaque. Arch Oral Biol. 2016;70:117-124.

doi:10.1016/j.archoralbio.2016.06.011.

Abdulbaqi HR, Himratul-Aznita WH, Baharuddin NA. Evaluation of Salvadora persica $L$. and green tea anti-plaque effect: a randomized controlled crossover clinical trial. BMC Complement Altern Med. 2016;16(1):7. doi:10.1186/s12906016-1487-0.

Abreu P, Matthew S, Gonzalez T, Costa D, Segundo MA, Fernandes E. Antiinflammatory and antioxidant activity of a medicinal tincture from Pedilanthus tithymaloides. Life Sciences. 2006;78(14):1578-1585.

doi:10.1016/j.Ifs.2005.07.037.
Not addressing research question

Not addressing research question

Not addressing research question

Not addressing research question

Allaker RP, Douglas CWI. Novel anti-microbial therapies for dental plaquerelated diseases. International Journal of Antimicrobial Agents. 2009;33(1):8-13. Not addressing doi:10.1016/j.ijantimicag.2008.07.014.

research question

Amurdhavani BS. Benefits of green tea in dentistry-a review. Res J Pharm Technol. 2015;8(6):772-774. doi:10.5958/0974-360X.2015.00124.9.

Review

Anonymous. Green tea and oral health examined in study. Br Dent J. 2010;208(9):384. doi:10.1038/sj.bdj.2010.436.

Not addressing research question

Arab H, Maroofian A, Golestani S, Shafaee H, Sohrabi K, Forouzanfar A. Review of The therapeutic effects of Camellia sinensis (green tea) on oral and periodontal health. Journal of Medicinal Plants Research. 2011;5(23):54655469.

Review

Araghizadeh A, Kohanteb J, Fani MM. Inhibitory activity of green tea (Camellia sinensis) extract on some clinically isolated cariogenic and periodontopathic bacteria. Med Princ Pract. 2013;22(4):368-372. doi:10.1159/000348299.

Not addressing research question

Ardakani MRT, Golmohammadi S, Ayremlou S, Taheri S, Daneshvar S, Meimandi M. Antibacterial Effect of Iranian Green-Tea-containing Mouthrinse vs Chlorhexidine 0.2\%: An In Vitro Study. Oral health prev. 2014;12(2):157-162.

Not addressing research question

Asahi $Y$, Noiri Y, Miura J, et al. Effects of the tea catechin epigallocatechin gallate on Porphyromonas gingivalis biofilms. J Appl Microbiol. 2014;116(5):1164-1171. doi:10.1111/jam.12458.

Not addressing research question

Avery MD. Current Resources for Evidence-Based Practice, May/June 2014. JOGNN: Journal of Obstetric, Gynecologic \& Neonatal Nursing. 2014;43(3):E22-91p. doi:10.1111/1552-6909.12314.

Not addressing research question

Awadalla HI, Ragab MH, Bassuoni MW, Fayed MT, Abbas MO. A pilot study of the role of green tea use on oral health. Int J Dent Hygiene. 2011;9(2):110-116. doi:10.1111/j.1601-5037.2009.00440.x.

Not addressing research question

Azelmat J, Larente JF, Grenier D. The anthraquinone rhein exhibits synergistic antibacterial activity in association with metronidazole or natural compounds and attenuates virulence gene expression in Porphyromonas gingivalis. Arch Oral Biol. 2015;60(2):342-346. doi:10.1016/j.archoralbio.2014.11.006.

Not addressing research question

Bai LL, Takagi S, Ando T, et al. Antimicrobial activity of tea catechin against canine oral bacteria and the functional mechanisms. J Vet Med Sci. 2016;78(9):1439-1445. doi:10.1292/jvms.16-0198.

Barilla J. Longevity. Healthy gums, healthy body: your gums are the key to a healthy smile, and more. Better Nutrition. 2000;62(3):24-262p.
Not addressing research question

Not addressing research question 
Bedran TBL, Morin MP, Spolidorio DP, Grenier D. Black tea extract and its theaflavin derivatives inhibit the growth of periodontopathogens and modulate interleukin-8 and $\beta$-defensin secretion in oral epithelial cells. PLOS ONE. 2015;10(11). doi:10.1371/journal.pone.0143158.

Ben Lagha A, Grenier D. Tea polyphenols inhibit the activation of NF-kappa B and the secretion of cytokines and matrix metalloproteinases by macrophages stimulated with Fusobacterium nucleatum. Sci Rep. 2016;6:11. doi:10.1038/srep34520.

Bioactive Foods in Promoting Health. Elsevier Inc.; 2010.

Cai L, Wu CD. Compounds from Syzygium aromaticum possessing growth inhibitory activity against oral pathogens. J NAT PROD. 1996;59(10):987-990. doi:10.1021/np960451q.

Cai Y, Chen Z, Liu H, Xuan Y, Wang X, Luan Q. Green tea epigallocatechin-3gallate alleviates Porphyromonas gingivalis-induced periodontitis in mice. Int Immunopharmacol. 2015;29(2):839-845. doi:10.1016/j.intimp.2015.08.033.

Cai Y, Chen Z, Liu H, Xuan Y, Wang X, Luan Q. Green tea epigallocatechin-3gallate alleviates Porphyromonas gingivalis-induced periodontitis in mice. Int Immunopharmacol. 2015;29(2):839-845. doi:10.1016/j.intimp.2015.08.033.

Chang HS, Hwang HJ, Kang EH, et al. The effect of green tea bag in dogs with periodontal disease. J Vet Clin. 2009;26(1):41-47.

Chatterjee A, Saluja M, Agarwal G, Alam M. Green tea: A boon for periodontal and general health. J Indian Soc Periodontol. 2012;16(2):161-167.

doi:10.4103/0972-124X.99256.

Chava VK, Vedula BD. Thermo-reversible green tea catechin gel for local

application in chronic periodontitis: a 4-week clinical trial. Journal of Periodontology. 2013;84(9):1290-1296. doi:10.1902/jop.2012.120425.

Chinsembu KC. Plants and other natural products used in the management of oral infections and improvement of oral health. Acta Trop. 2016;154:6-18. doi:10.1016/j.actatropica.2015.10.019.

Cho AR, Kim JH, Lee DE, et al. The effect of orally administered epigallocatechin-3-gallate on ligature-induced periodontitis in rats. $J$ Periodontal Res. 2013;48(6):781-7899p. doi:10.1111/jre.12071.

Not addressing research question

Chopra A, Thomas BS, Sivaraman K, Prasad HK, Kamath SU. Green Tea Intake as an Adjunct to Mechanical Periodontal Therapy for the Management of Mild to Moderate Chronic Periodontitis: A Randomized Controlled Clinical Trial. Oral health prev. 2016;14(4):293-303. doi:10.3290/j.ohpd.a36100.

Not addressing research question

Clutterbuck AL, Asplin KE, Harris P, Allaway D, Mobasheri A. Targeting matrix metalloproteinases in inflammatory conditions. Curr Drug Targets. 2009;10(12):1245-1254. doi:10.2174/138945009789753264.

Not addressing research question

Counteraction to Chemical and Biological Terrorism in East European Countries. Dordrecht: Springer; 2009.

Coutts A. Clinical. Nutrition and the life cycle 4: the healthy diet for the adult. British Journal of Nursing. 2001;10(6):362-3697p.

Current Trends in Periodontics and Implant Dentistry. Nova Science Publishers, Inc.; 2013.

D'Dharan SR, Neelakantan P. Therapeutic uses of cranberry (Vaccinium macrocarpon) extract - A review. Intl J Pharmacogn Phytochem Res. 2013;5(3):197-199.

Not addressing research question

Not addressing research question

Not addressing research question

Not addressing research question

Da Silva APB, Bissada NF. Genetic and behavioral risk factors associated with periodontal disease. In: Current Trends in Periodontics and Implant Dentistry. Nova Science Publishers, Inc.; 2013:41-59.

Not addressing research question

de Oliveira JS, Pinto M, Santana LDD, Pinto ASB, di Lenardo D, Vasconcelos DFP. Biological Effects of Medicinal Plants on Induced Periodontitis: A Systematic Review. Int J Dent. 2016:10. doi:10.1155/2016/3719879.
Not addressing research question 
De Sá Siqueira MA, Fischer RG, Figueredo CMDS, Brunini TMC, MendesRibeiro AC. Nitric oxide and oral diseases: Can we talk about it? cardiovasc Hematol Agents Med Chem. 2010;8(2):104-112.

Not addressing doi:10.2174/187152510791170942. research question

Desjardins J, Grenier D. Neutralizing effect of green tea epigallocatechin-3gallate on nicotine-induced toxicity and chemokine (C-C motif) ligand 5 secretion in human oral epithelial cells and fibroblasts. J Investig Clin Dent. Not addressing 2012;3(3):189-197. doi:10.1111/j.2041-1626.2011.00103.x. research question

Dumitrescu AL. Exploring the relationship between nutrition and periodontal disease. In: Oral Health: Anesthetic Management, Social Determinants, Role of Nutrition and Impact on Quality of Life. Nova Science Publishers, Inc.; 2015:6590.

Not addressing research question

Elnaggar WA, Taha TH, El-Deeb NM, Arafat HH. Efficacy of non-cytotoxic doses of some medicinal plant extracts as antibacterial and anti-biofilm agents against cariogenic bacterium streptococcus mutans. Biosci Biotechnol Res Asia. 2016;13(2):1279-1284. doi:10.13005/bbra/2163.

Not addressing research question Not addressing research question

Erickson K. Tea time. Better Nutrition. 2008;70(10):38-[40]2p.

Not addressing research question

Not addressing research question

Cambridge: Woodhead Publ Ltd; 2009.

Fournier-Larente J, Morin MP, Grenier D. Green tea catechins potentiate the effect of antibiotics and modulate adherence and gene expression in Porphyromonas gingivalis. Arch Oral Biol. 2016;65:35-43. doi:10.1016/j.archoralbio.2016.01.014.

Not addressing research question

Funosas ER, Martínez AB, Pignolo M, et al. Efficacy of green tea in the treatment of chronic periodontitis. Av Odontoestomatol. 2005;21(3):159-166.

Funosas ER, Martinez AB, Pignolo M, Maestri L, Lucena PSH. Green tea subgingival gel effectiveness in chronic periodontitis. Journal of Dental Research. 2003;82:18-18.

full text assessment conference paper/duplicate $\mathrm{N}^{\circ} 63 /$ paper requested

Gadagi JS, Chava VK, Reddy VR. Green tea extract as a local drug therapy on periodontitis patients with diabetes mellitus: A randomized case-control study. $J$ Indian Soc Periodontol. 2013;17(2):198-203. doi:10.4103/0972-124X.113069.

full text assessment

Gaur S, Agnihotri R. Green tea: a novel functional food for the oral health of older adults. Geriatrics \& Gerontology International. 2014;14(2):238-250. doi:10.1111/ggi.12194.

Review

Gennaro G, Claudino M, Cestari TM, et al. Green Tea Modulates Cytokine Expression in the Periodontium and Attenuates Alveolar Bone Resorption in Type 1 Diabetic Rats. PLoS ONE. 2015;10(8):e0134784. doi:10.1371/journal.pone.0134784.

Gennaro G, Claudino M, Cestari TM, et al. Green Tea Modulates Cytokine Expression in the Periodontium and Attenuates Alveolar Bone Resorption in Type 1 Diabetic Rats. PLoS ONE. 2015;10(8):e0134784. doi:10.1371/journal.pone.0134784.

Not addressing research question

Govindaraj J, Emmadi P, Puvanakrishnan R. Therapeutic effects of proanthocyanidins on the pathogenesis of periodontitis-An overview. Indian Journal of Experimental Biology. 2011;49(2):83-93.

Not addressing research question

Granica S, Kłẹbowska A, Kosiński M, et al. Effects of Geum urbanum L. root extracts and its constituents on polymorphonuclear leucocytes functions. Significance in periodontal diseases. J Ethnopharmacol. 2016;188:1-12. doi:10.1016/j.jep.2016.04.030.

Green is good for your teeth. Explorer (08947929). 2011;37(3):4-41p.

Not addressing research question 
Grenier D, La VD. Proteases of Porphyromonas gingivalis as important virulence factors in periodontal disease and potential targets for plant-derived compounds: a review article. Curr Drug Targets. 2011;12(3):322-331.

Not addressing Groppo FC, De Cássia Bergamaschi C, Cogo K, Franz-Montan M, Motta RHL, De Andrade ED. Use of phytotherapy in dentistry. Phytother Res. 2008;22(8):993-998. doi:10.1002/ptr.2471. research question

Not addressing research question

Gurav AN. Advanced glycation end products: A link between periodontitis and diabetes mellitus? Curr Diabetes Rev. 2013;9(5):355-361.

doi:10.2174/15733998113099990066.

Not addressing

research question

Gurenlian JR, Spolarich AE. Risk assessment for clients with diabetes. Access. Not addressing 2010;24(6):32-354p.

research question

Gutierrez-Venegas G, Contreras-Sanchez A. Luteolin and fisetin inhibit the effects of lipopolysaccharide obtained from Porphyromonas gingivalis in human gingival fibroblasts. Molecular Biology Reports. 2013;40(1):477-485.

Not addressing doi:10.1007/s11033-012-2083-0. research question

Hamer M. The beneficial effects of tea on immune function and inflammation: a review of evidence from in vitro, animal, and human research. Nutr Res.

Not addressing 2007;27(7):373-379. doi:10.1016/j.nutres.2007.05.008.

research question

Han K, Hwang E, Park JB. Association between Consumption of Coffee and the Prevalence of Periodontitis: The 2008-2010 Korea National Health and Nutrition Examination Survey. PLOS ONE. 2016;11(7):11. doi:10.1371/journal.pone.0158845.

Han K, Hwang E, Park JB. Excessive Consumption of Green Tea as a Risk Factor for Periodontal Disease among Korean Adults. Nutrients. 2016;8(7):02. doi:10.3390/nu8070408.

Not addressing research question

Not addressing research question

Hattarki SA, Pushpa SP, Bhat K. Evaluation of the efficacy of green tea catechins as an adjunct to scaling and root planing in the management of chronic periodontitis using PCR analysis: A clinical and microbiological study. $J$ Indian Soc Periodontol. 2013;17(2):204-209. doi:10.4103/0972-124X.113071.

Full text assessment

Hirasawa M, Takada K, Makimura M, Otake S. Improvement of periodontal status by green tea catechin using a local delivery system: a clinical pilot study. J Periodontal Res. 2002;37(6):433-438.

Homer KA, Manji F, Beighton D. INHIBITION OF PEPTIDASE AND

full text assessment

GLYCOSIDASE ACTIVITIES OF PORPHYROMONAS-GINGIVALIS,

BACTEROIDES-INTERMEDIUS AND TREPONEMA-DENTICOLA BY PLANT-

EXTRACTS. Journal of Clinical Periodontology. 1992;19(5):305-310. doi:10.1111/j.1600-051X.1992.tb00649.x.

Not addressing

research question

Hong JY, Yon J, Lee JS, et al. Effects of epigallocatechin-3-gallate on the

healing of extraction sockets with a periapical lesion: A pilot study in dogs. Journal of Biomedical Materials Research Part B-Applied Biomaterials. 2015;103(4):727-734. doi:10.1002/jbm.b.33238.

Hosokawa Y, Hosokawa I, Ozaki K, Nakanishi T, Nakae H, Matsuo T. Catechins inhibit CCL20 production in IL-17A-stimulated human gingival fibroblasts. Cell Physiol Biochem. 2009;24(5-6):391-396. doi:10.1159/000257431.

Hosokawa Y, Hosokawa I, Ozaki K, Nakanishi T, Nakae H, Matsuo T. Catechins inhibit CXCL10 production from oncostatin M-stimulated human gingival fibroblasts. J nutr biochem. 2010;21(7):659-664. doi:10.1016/j.jnutbio.2009.04.005.

Not addressing research question

Hosokawa Y, Hosokawa I, Ozaki K, Nakanishi T, Nakae H, Matsuo T. Tea polyphenols inhibit IL-6 production in tumor necrosis factor superfamily 14stimulated human gingival fibroblasts. Mol Nutr Food Res. 2010;54 Suppl 2:S151-S158. doi:10.1002/mnfr.200900549.

Not addressing research question

Hosokawa Y, Hosokawa I, Shindo S, et al. Black tea polyphenol inhibits CXCL10 production in oncostatin M-stimulated human gingival fibroblasts. Int Immunopharmacol. 2011;11(6):670-674. doi:10.1016/j.intimp.2011.01.009.

Not addressing research question

Not addressing research question

Not addressing research question 
Hosokawa Y, Hosokawa I, Shindo S, Ozaki K, Matsuo T. (-)-Epigallocatechin-3gallate inhibits $\mathrm{CC}$ chemokine ligand 11 production in human gingival

Not addressing fibroblasts. Cell Physiol Biochem. 2013;31(6):960-967. doi:10.1159/000350114. research question Houde V, Grenier D, Chandad F. Protective effects of grape seed proanthocyanidins against oxidative stress induced by lipopolysaccharides of periodontopathogens. Journal of Periodontology. 2006;77(8):1371-1379. doi:10.1902/jop.2006.050419.

Not addressing research question

Hrishi T, Kundapur P, Naha A, Thomas B, Kamath S, Bhat G. Effect of adjunctive use of green tea dentifrice in periodontitis patients - A Randomized Controlled Pilot Study. Int J Dent Hygiene. 2015. doi:10.1111/idh.12131.

Not addressing research question

Hrishi TS, Kundapur PP, Naha A, Thomas BS, Kamath S, Bhat GS. Effect of adjunctive use of green tea dentifrice in periodontitis patients - A Randomized Controlled Pilot Study. Int J Dent Hygiene. 2016;14(3):178-183. doi:10.1111/idh.12131.

Not addressing research question

In the public eye: what your patients are reading in consumer magazines,

Not addressing newspapers, and on Web sites. RN. 2007;70(1):3-31p.

research question

Inaba $\mathrm{H}$, Tagashira $\mathrm{M}$, Honma $\mathrm{D}$, et al. Identification of hop polyphenolic components which inhibit prostaglandin E2 production by gingival epithelial cells stimulated with periodontal pathogen. Biol Pharm Bull. 2008;31(3):527-530.

Not addressing Inaba H, Tagashira M, Kanda T, Amano A. Proliferation of Smooth Muscle Cells Stimulated by Porphyromonas Gingivalis is Inhibited by Apple Polyphenol. Journal of Periodontology. 2011;82(11):1616-1622. doi:10.1902/jop.2011.100785. research question

Inaba H, Tagashira M, Kanda T, Murakami Y, Amano A, Matsumoto-Nakano M. Apple and hop-polyphenols inhibit porphyromonas gingivalis-mediated precursor of matrix metalloproteinase-9 activation and invasion of oral squamous cell carcinoma cells. Journal of Periodontology. 2016;87(9):11031111. doi:10.1902/jop.2016.160047.

Inaba H, Tagashira M, Kanda T, Murakami Y, Amano A, Matsumoto-Nakano M. Apple- and Hop-Polyphenols Inhibit Porphyromonas gingivalis-Mediated Precursor of Matrix Metalloproteinase-9 Activation and Invasion of Oral Squamous Cell Carcinoma Cells. Journal of Periodontology. 2016;87(9):11031111. doi:10.1902/jop.2016.160047.

Not addressing research question

Isogai $\mathrm{E}$, Isogai $\mathrm{H}$, Kimura K, Nishikawa T, Fujii N, Benno Y. EFFECT OF JAPANESE GREEN TEA EXTRACT ON CANINE PERIODONTAL-DISEASES. Microbial Ecology in Health and Disease. 1995;8(2):57-61. Isogai $\mathrm{H}$, Isogai $\mathrm{E}$, Takahashi K, Kurebayashi Y. Effect of catechin diet on gingivitis in cats. International Journal of Applied Research in Veterinary Medicine. 2008;6(2):82-86.

Not addressing research question

Iwaki K, Koya-Miyata S, Kohno K, Ushio S, Fukuda S. Antimicrobial activity of Polygonum tinctorium Lour: Extract against oral pathogenic bacteria. J Nat Med. Not addressing 2006;60(2):121-125. doi:10.1007/s11418-005-0025-z.

Izui S, Sekine S, Maeda K, et al. Antibacterial Activity of Curcumin Against Periodontopathic Bacteria. Journal of Periodontology. 2016;87(1):83-90. doi:10.1902/jop.2015.150260. research question

Not addressing research question

Izui S, Sekine S, Maeda K, et al. Antibacterial Activity of Curcumin Against Periodontopathic Bacteria. Journal of Periodontology. 2016;87(1):83-90. doi:10.1902/jop.2015.150260.

Jenabian N, Moghadamnia AA, Karami E, Mir AP. The effect of Camellia Sinensis (green tea) mouthwash on plaque-induced gingivitis: a single-blinded randomized controlled clinical trial. Daru. 2012;20(1):39. doi:10.1186/20082231-20-39.

Johnson BJ, Lin B, Bongard JE. Genus Vaccinium: Medicine, cosmetics, and coatings. Recent Pat Biotechnol. 2010;4(2):112-124. doi:10.2174/187220810791110732. Johnston BD, Fritz, P. C., Ward WE. Use of dietary supplements in patients seeking treatment at a periodontal clinic. Nutrients. 2013;5(4):1110-1121. doi:10.3390/nu5041110.

In vitro study

Not addressing research question

Not addressing research question

Not addressing research question

Not addressing research question

Not addressing research question

Not addressing research question 
Jung IH, Lee DE, Yun JH, et al. Anti-inflammatory effect of (-)-epigallocatechin3-gallate on Porphyromonas gingivalis lipopolysaccharide-stimulated fibroblasts and stem cells derived from human periodontal ligament. $J$ Periodontal Implant Sci. 2012;42(6):185-195. doi:10.5051/jpis.2012.42.6.185.

Jurenka J. Therapeutic applications of pomegranate (Punica granatum L.): a review. Alternative Medicine Review. 2008;13(2):128-14417p.

Kang MS, Jang HS, Oh JS, et al. Effects of methyl gallate and gallic acid on the production of inflammatory mediators interleukin- 6 and interleukin-8 by oral epithelial cells stimulated with Fusobacterium nucleatum. J. 2009;47(6):760767. doi:10.1007/s12275-009-0097-7.
Not addressing research question

Not addressing research question

Not addressing research question

Kang MS, Jang HS, Oh JS, et al. Effects of methyl gallate and gallic acid on the production of inflammatory mediators interleukin- 6 and interleukin-8 by oral epithelial cells stimulated with Fusobacterium nucleatum. J. 2010;47(6):760767. doi:10.1007/s12275-009-0097-7.

Kang MS, Oh JS, Kang IC, Hong SJ, Choi CH. Inhibitory effect of methyl gallate and gallic acid on oral bacteria. J. 2008;46(6):744-750. doi:10.1007/s12275008-0235-7.

Not addressing research question

Not addressing research question

Kanzaki H, Shinohara F, Itohiya-Kasuya K, Ishikawa M, Nakamura Y. Nrf2 activation attenuates both orthodontic tooth movement and relapse. J Dent Res. 2015;94(6):787-794. doi:10.1177/0022034515577814.

Kara M, Kesim S, Aral CA, Elmali F. EFFECT OF GRAPE SEED EXTRACT UPON PLASMA OXIDATIVE STATUS AND ALVEOLAR BONE, IN LIGATURE INDUCED PERIODONTITIS. Biotechnology \& Biotechnological Equipment. 2013;27(5):4131-4136. doi:10.5504/bbeq.2013.0074.

Not addressing research question

Karygianni L, Al-Ahmad A, Argyropoulou A, Hellwig E, Anderson AC, Skaltsounis AL. Natural Antimicrobials and Oral Microorganisms: A Systematic Review on Herbal Interventions for the Eradication of Multispecies Oral Biofilms. Front Microbiol. 2015;6:1529. doi:10.3389/fmicb.2015.01529.

Not addressing research question

Karygianni L, Al-Ahmad A, Argyropoulou A, Hellwig E, Anderson AC, Skaltsounis AL. Natural Antimicrobials and Oral Microorganisms: A Systematic Review on Herbal Interventions for the Eradication of Multispecies Oral Biofilms. Front Microbiol. 2016;6:17. doi:10.3389/fmicb.2015.01529.

Not addressing research question

Kaur G, Kathariya R, Bansal S, Singh A, Shahakar D. Dietary antioxidants and their indispensable role in periodontal health. J Food Drug Anal. 2016;24(2):239-246. doi:10.1016/j.jfda.2015.11.003.

Not addressing research question

Kaur H, Jain S, Kaur A. Comparative evaluation of the antiplaque effectiveness of green tea catechin mouthwash with chlorhexidine gluconate. J Indian Soc Periodontol. 2014;18(2):178-182. doi:10.4103/0972-124X.131320.

Kelly GS. Quercetin. Alternative Medicine Review. 2011;16(2):172-19423p. Kemoli AM, van Amerongen WE, de Soet JJ. Antimicrobial and buffer capacity of crude extracts of chewing sticks (Miswaki) from Kenya. J Dent Child. 2001;68(3):183-8-152.

Not addressing research question Not addressing research question

Not addressing research question

Khurshid Z, Zafar MS, Zohaib S, Najeeb S, Naseem M. Green Tea (Camellia Sinensis): Chemistry and Oral Health. Open Dent J. 2016;10:166-173. doi:10.2174/1874210601610010166.

Kiefer D. Disease prevention begins in the mouth. Life Extension. 2008;14(9):28-3710p.

Kim-Park WK, Allam ES, Palasuk J, Kowolik M, Park KK, Windsor LJ. Green tea catechin inhibits the activity and neutrophil release of Matrix Metalloproteinase-9. J. 2016;6(4):343-346.

Kim-Park WK, Allam ES, Palasuk J, Kowolik M, Park KK, Windsor LJ. Green tea catechin inhibits the activity and neutrophil release ofMatrix Metalloproteinase-9. J Tradit Complement Med. 2015. doi:10.1016/j.jtcme.2015.02.002.
Not addressing research question Not addressing research question

Not addressing

Not addressing research question research question 
Kong LX, Qi X, Huang SY, Chen SS, Wu YF, Zhao L. Theaflavins inhibit pathogenic properties of $P$. gingivalis and MMPs production in $P$. gingivalisstimulated human gingival fibroblasts. Arch Oral Biol. 2015;60(1):12-22. doi:10.1016/j.archoralbio.2014.08.019.

Not addressing

Kou Y, Inaba H, Kato T, et al. Inflammatory responses of gingival epithelial cells stimulated with Porphyromonas gingivalis vesicles are inhibited by hopassociated polyphenols. Journal of Periodontology. 2008;79(1):174-180. doi:10.1902/jop.2008.070364.

research question

Kouidhi B, Qurashi AI YMA, Chaieb K. Drug resistance of bacterial dental biofilm and the potential use of natural compounds as alternative for prevention and treatment. Microbial Pathogenesis. 2015;80:39-49. doi:10.1016/j.micpath.2015.02.007.

Not addressing research question

Not addressing research question

Koyama Y, Kuriyama S, Aida J, et al. Association between green tea consumption and tooth loss: Cross-sectional results from the Ohsaki Cohort 2006 Study. Prev Med. 2010;50(4):173-179. doi:10.1016/j.ypmed.2010.01.010.

Not addressing research question

Kudva P, Tabasum ST, Shekhawat NK. Effect of green tea catechin, a local drug delivery system as an adjunct to scaling and root planing in chronic periodontitis patients: A clinicomicrobiological study. J Indian Soc Periodontol. 2011;15(1):39-45. doi:10.4103/0972-124X.82269.

full text assessment

Kukreja BJ, Dodwad V. Herbal mouthwashes - A gift of nature. Intl J Pharma Bio Sci. 2012;3(2):P46-P52.

Not addressing research question

Kurita-Ochiai T, Jia R, Cai Y, Yamaguchi Y, Yamamoto M. Periodontal DiseaseInduced Atherosclerosis and Oxidative Stress. Antioxidants. 2015;4(3):577-590. doi:10.3390/antiox4030577.

Not addressing research question

Kushiyama M, Shimazaki Y, Murakami M, Yamashita Y. Relationship between intake of green tea and periodontal disease. Journal of Periodontology. 2009;80(3):372-377. doi:10.1902/jop.2009.080510.

Laine ML, Crielaard W. Functional foods/ingredients and periodontal diseases. European Journal of Nutrition. 2012;51:27-30. doi:10.1007/s00394-012-0325-5.

Lauten JD, Boyd L, Hanson MB, Lillie D, Gullion C, Madden TE. A clinical study: Melaleuca, Manuka, Calendula and green tea mouth rinse. Phytother Res. 2005;19(11):951-957. doi:10.1002/ptr.1763.

Not addressing research question

Not addressing research question

Lee B-S, Lee C-C, Lin H-P, et al. A functional chitosan membrane with grafted epigallocatechin-3-gallate and lovastatin enhances periodontal tissue regeneration in dogs. Carbohydrate polymers. 2016;151:790-802. doi:10.1016/j.carbpol.2016.06.026.

Lee R, Bayne A, Tiangco M, Garen G, Chow A. Prevention of tea-induced extrinsic tooth stain. Int J Dent Hygiene. 2014;12(4):267-2726p. doi:10.1111/idh.12096.

Not addressing research question

Lee YL, Hong CY, Kok SH, et al. An extract of green tea, epigallocatechin-3gallate, reduces periapical lesions by inhibiting cysteine-rich 61 expression in osteoblasts. J Endod. 2009;35(2):206-211. doi:10.1016/j.joen.2008.11.015. Lerman A, Lockwood B. Nutraceuticals in veterinary medicine. Pharm J. 2007;278(7434):51-55.

Not addressing research question

Levy Y. Oxidative stress, antioxidants and periodontal disease. Arch Oral Biol. 2015;60(9):1461-1462. doi:10.1016/j.archoralbio.2015.07.015.

Not addressing research question

Li NG, Shi ZH, Tang YP, Duan JA. Selective matrix metalloproteinase inhibitors for cancer. Curr Med Chem. 2009;16(29):3805-3827. doi:10.2174/092986709789178037.

Not addressing research question

Not addressing research question

Not addressing research question

Not addressing research question

Lindinger MI. Reduced Dental Plaque Formation in Dogs Drinking a Solution Containing Natural Antimicrobial Herbal Enzymes and Organic Matcha Green Tea. Scientifica (Cairo). 2016;2016:2183623.

Lingström P, Zaura E, Hassanö H, et al. The Anticaries Effect of a Food Extract (Shiitake) in a Short-Term Clinical Study. J Biomed Biotechnol. 2012;2012:11010p.

Not addressing research question

Not addressing research question 
Logan El. Dietary Influences on Periodontal Health in Dogs and Cats. Vet Clin North Am Small Anim Pract. 2006;36(6):1385-1401.

doi:10.1016/j.cvsm.2006.09.002.

Not addressing

Lohr G, Beikler T, Podbielski A, Standar K, Redanz S, Hensel A. Polyphenols

research question

from Myrothamnus flabellifolia Welw. inhibit in vitro adhesion of Porphyromonas gingivalis and exert anti-inflammatory cytoprotective effects in KB cells. Journal of Clinical Periodontology. 2011;38(5):457-469. doi:10.1111/j.1600-

051X.2010.01654.x.

Lombardo Bedran TB, Feghali K, Zhao L, Palomari Spolidorio DM, Grenier D. Green tea extract and its major constituent, epigallocatechin-3-gallate, induce epithelial beta-defensin secretion and prevent beta-defensin degradation by Porphyromonas gingivalis. J Periodontal Res. 2014;49(5):615-623.

doi:10.1111/jre.12142.

Not addressing
research question

Lombardo Bedran TB, Morin MP, Palomari Spolidorio D, Grenier D. Black Tea Extract and Its Theaflavin Derivatives Inhibit the Growth of

Periodontopathogens and Modulate Interleukin- 8 and beta-Defensin Secretion in Oral Epithelial Cells. PLoS ONE. 2015;10(11):e0143158.

doi:10.1371/journal.pone.0143158.

Lombardo Bedran TB, Morin MP, Palomari Spolidorio D, Grenier D. Black Tea

Extract and Its Theaflavin Derivatives Inhibit the Growth of

Periodontopathogens and Modulate Interleukin-8 and beta-Defensin Secretion in Oral Epithelial Cells. PLOS ONE. 2015;10(11):e0143158.

doi:10.1371/journal.pone.0143158.

Not addressing

research question

Lombardo Bedran TB, Palomari Spolidorio D, Grenier D. Green tea polyphenol epigallocatechin-3-gallate and cranberry proanthocyanidins act in synergy with cathelicidin (LL-37) to reduce the LPS-induced inflammatory response in a three-dimensional co-culture model of gingival epithelial cells and fibroblasts. Arch Oral Biol. 2015;60(6):845-853. doi:10.1016/j.archoralbio.2015.02.021.

Not addressing

research question

Not addressing

research question

Lombardo Bedran TB, Palomari Spolidorio D, Grenier D. Green tea polyphenol epigallocatechin-3-gallate and cranberry proanthocyanidins act in synergy with cathelicidin (LL-37) to reduce the LPS-induced inflammatory response in a three-dimensional co-culture model of gingival epithelial cells and fibroblasts. Arch Oral Biol. 2015;60(6):845-853. doi:10.1016/j.archoralbio.2015.02.021.

Not addressing Low SB, Peak RM, Smithson CW, Perrone J, Gaddis B, Kontogiorgos E. Evaluation of a topical gel containing a novel combination of essential oils and antioxidants for reducing oral malodor in dogs. American Journal of Veterinary Research. 2014;75(7):653-657.

research question

Not addressing research question

Ma R, Sun M, Huang L, et al. Effect of compound Chinese traditional medicine on periapical periodontitis in dogs and cytotoxic assay. Shanghai Kou Qiang Yi Xue. 2012;21(6):643-647.

Machida T, Tomofuji T, Ekuni D, et al. Severe Periodontitis Is Inversely Associated with Coffee Consumption in the Maintenance Phase of Periodontal Treatment. Nutrients. 2014;6(10):4476-4490. doi:10.3390/nu6104476.

Not addressing research question

Maeda-Yamamoto M. Human clinical studies of tea polyphenols in allergy or life style-related diseases. Curr Pharm Des. 2013;19(34):6148-6155. doi:10.2174/1381612811319340009.

Not addressing research question

Not addressing research question

Magee $\mathrm{K}$, Loiacono C. A review of common herbs and potential interactions. Int J Dent Hygiene. 2004;2(3):111-12111p.

Not addressing research question

Mahyari S, Mahyari B, Emami SA, et al. Evaluation of the efficacy of a polyherbal mouthwash containing Zingiber officinale, Rosmarinus officinalis and Calendula officinalis extracts in patients with gingivitis: A randomized doubleblind placebo-controlled trial. Complement Ther Clin Pract. 2016;22:93-98. doi:10.1016/j.ctcp.2015.12.001.

Not addressing research question

Makimura M, Hirasawa M, Kobayashi K, et al. Inhibitory effect of tea catechins on collagenase activity. Journal of Periodontology. 1993;64(7):630-636. Mandal M, Mandal A, Das S, Chakraborti T, Chakraborti S. Clinical implications of matrix metalloproteinases. Mol Cell Biochem. 2003;252(1-2):305-329. doi:10.1023/A:1025526424637.

Not addressing research question

Not addressing research question

Not addressing research question 
Maruyama T, Tomofuji T, Endo Y, et al. Supplementation of green tea catechins in dentifrices suppresses gingival oxidative stress and periodontal inflammation. Arch Oral Biol. 2011;56(1):48-53. doi:10.1016/j.archoralbio.2010.08.015.

Not addressing Matsunaga T, Nakahara A, Minnatul KM, et al. The Inhibitory Effects of Catechins on Biofilm Formation by the Periodontopathogenic Bacterium, Eikenella corrodens. Bioscience Biotechnology and Biochemistry. 2010;74(12):2445-2450. doi:10.1271/bbb.100499.

Mays RM, Gordon RA, Wilson JM, Silapunt S. New antibiotic therapies for acne and rosacea. Dermatol Ther. 2012;25(1):23-37. doi:10.1111/j.15298019.2012.01497.x. research question

McKay DL, Blumberg JB. Cranberries (Vaccinium macrocarpon) and cardiovascular disease risk factors. Nutrition Reviews. 2007;65(11):49050213p.

Not addressing research question

Not addressing research question

Miller AL. Antioxidant flavonoids: structure, function and clinical usage. Alternative Medicine Review. 1996;1(2):103-1119p.

Not addressing research question

Not addressing research question

Morin MP, Bedran TBL, Fournier-Larente J, Haas B, Azelmat J, Grenier D. Green tea extract and its major constituent epigallocatechin-3-gallate inhibit growth and halitosis-related properties of Solobacterium moorei. Bmc Complementary and Alternative Medicine. 2015;15:11. doi:10.1186/s12906015-0557-z.

Not addressing research question

Morin MP, Grenier D. Regulation of matrix metalloproteinase secretion by green tea catechins in a three-dimensional co-culture model of macrophages and gingival fibroblasts. Arch Oral Biol. November 2016. doi:10.1016/j.archoralbio.2016.10.035.

Not addressing research question

Murphy K, Beliak O, Shaw A. Reviews of articles on medicinal herbs. Australian Not addressing Journal of Herbal Medicine. 2012;24(4):147-1504p.

research question

Nagata $\mathrm{H}$, Inagaki $\mathrm{Y}$, Yamamoto $\mathrm{Y}$, et al. Inhibitory effects of macrocarpals on the biological activity of Porphyromonas gingivalis and other periodontopathic bacteria. Oral Microbiol Immunol. 2006;21(3):159-163. doi:10.1111/j.1399302X.2006.00269.x.

Nakatsuka Y, Nagasawa T, Yumoto Y, Nakazawa F, Furuichi Y. Inhibitory effects of sword bean extract on alveolar bone resorption induced in rats by Porphyromonas gingivalis infection. J Periodontal Res. 2014;49(6):801-809. doi:10.1111/jre.12166.

News from The Journal of Chinese Medicine. Journal of Chinese Medicine. 2006;(81):67-737p.

Niloofar J, Ali AM, Elaheh K, Poorsattar BMA. The effect of Camellia Sinensis (green tea) mouthwash on plaque-induced gingivitis: a single-blinded randomized controlled clinical trial. Daru. 2012;20:6. doi:10.1186/2008-2231-2039.

Ninger LJ. Green tea helps prevent periodontal disease. Life Extension. 2009;15(7):21-211p.

Noguchi K, Endo $\mathrm{H}$, Kondo $\mathrm{H}$, Ishikawa I. Prostaglandin F2a upregulates interleukin-6 production in human gingival fibroblasts. J Periodontal Res. 2001;36(2):80-87.

Not addressing research question

Not addressing research question Not addressing research question

Nugala B, Namasi A, Emmadi P, Krishna PM. Role of green tea as an antioxidant in periodontal disease: The Asian paradox. $J$ Indian Soc Periodontol. 2012;16(3):313-316. doi:10.4103/0972-124X.100902.

Oberg E. Preconception counseling: helping patients plan for the future. Integrative Medicine: A Clinician's Journal. 2009;8(4):46-494p.

Okamoto M, Sugimoto A, Leung K-P, Nakayama K, Kamaguchi A, Maeda N. Inhibitory effect of green tea catechins on cysteine proteinases in Porphyromonas gingivalis. Oral Microbiol Immunol. 2004;19(2):118-120. doi:10.1046/j.0902-0055.2003.00112.x.

Opatrná $\mathrm{S}$. Is it possible to decrease microinflammation in chronic kidney disease patients? Akt Nefrol. 2015;21(2):43-48.

Not addressing research question Not addressing research question

Not addressing research question

Oral Health: Anesthetic Management, Social Determinants, Role of Nutrition and Impact on Quality of Life. Nova Science Publishers, Inc.; 2015.

Review

Not addressing research question

Not addressing research question

Not addressing research question

Not addressing research question 
Palaska I, Papathanasiou E, Theoharides TC. Use of polyphenols in periodontal inflammation. European Journal of Pharmacology. 2013;720(1-3):77-83. doi:10.1016/j.ejphar.2013.10.047.

Pandit S, Song K-Y, Jeon J-G. Withania somnifera Attenuates Acid Production, Acid Tolerance and Extra-Cellular Polysaccharide Formation of Streptococcus mutans Biofilms. American Journal of Chinese Medicine. 2014;42(1):15717115p. doi:10.1142/S0192415X14500116.

Park KM, You JS, Lee HY, Baek NI, Hwang JK. Kuwanon G: An antibacterial agent from the root bark of Morus alba against oral pathogens. $J$ Ethnopharmacol. 2003;84(2-3):181-185. doi:10.1016/S0378-8741(02)00318-5.

Parnham MJ. Immunomodulatory approaches to the treatment of infections. Infektol Glas. 2011;31(1):15-27.

Pasternak B, Aspenberg P. Metalloproteinases and their inhibitors -- diagnostic and therapeutic opportunities in orthopedics. Acta Orthopaedica. 2009;80(6):693-70412p. doi:10.3109/17453670903448257.

Patel VK, Venkatakrishna-Bhatt $\mathrm{H}$. Folklore therapeutic indigenous plants in periodontal disorders in India (review, experimental and clinical approach). Int $J$ Clin Pharmacol Ther Toxicol. 1988;26(4):176-184.

Patil AG, Jobanputra AH. Formulation, characterization and evaluation of a thermoresponsive in situ gel containing gallic acid-loaded chitosan nanoparticles for the treatment of periodontal disease. J Bionanoscience. 2015;9(5):401-408. doi:10.1166/jbns.2015.1324.

Pavel L, Pavel S. [Usefulness of micronutrients in the treatment of periodontitis]. Ned Tijdschr Tandheelkd. 2010;117(2):103-106.

Not addressing research question

Not addressing research question

Not addressing research question

Not addressing research question

Not addressing research question

Not addressing research question

Not addressing research question

Not addressing research question

Prabhakar J, Senthilkumar M, Priya MS, Mahalakshmi K, Sehgal PK,

Sukumaran VG. Evaluation of Antimicrobial Efficacy of Herbal Alternatives (Triphala and Green Tea Polyphenols), MTAD, and 5\% Sodium Hypochlorite against Enterococcus faecalis Biofilm Formed on Tooth Substrate: An In Vitro Study. J Endod. 2010;36(1):83-86. doi:10.1016/j.joen.2009.09.040.

Not addressing research question

Prithi R, Geetha RV. Static effects of fruits on periodontitis. Res J Pharm Technol. 2014;7(3):365-367.

Not addressing research question

Priya BM, Anitha V, Shanmugam M, Ashwath B, Sylva SD, Vigneshwari SK. Efficacy of chlorhexidine and green tea mouthwashes in the management of dental plaque-induced gingivitis: A comparative clinical study. Contemp. 2015;6(4):505-509. doi:10.4103/0976-237X.169845.

Not addressing research question

Radler DR. Nutritional supplements, ergogenic aids, and herbals. Dent Clin Not addressing North Am. 2003;47(2):245-258. doi:10.1016/S0011-8532(02)00099-X. research question

Rajini Kanth M, Ravi Prakash A, Sreenath G, Reddy VS, Huldah S. Efficacy of specific plant products on dental caries causing microorganisms. J Clin Diagn Res. 2016;10(12):ZM01-ZM03. doi:10.7860/JCDR/2016/19772.9025.

Not addressing research question

Ramasamy C. "Potential natural antioxidants: adjuvant effect of green tea polyphenols in periodontal infections." Infect Disord Drug Targets.

2015;15(3):141-152.

Review

Ramasamy C. Potential natural antioxidants: adjuvant effect of green tea polyphenols in periodontal infections. Infect Disord Drug Targets.

2015;15(3):141-152.

Review

Ramesh A, Varghese SS, Doraiswamy JN, Malaiappan S. Herbs as an antioxidant arsenal for periodontal diseases. J Intercult Ethnopharmacol. 2016;5(1):92-96. doi:10.5455/jice.20160122065556.

Not addressing Rattanasuwan K, Rassameemasmaung S, Sangalungkarn V, Komoltri C. research question Clinical effect of locally delivered gel containing green tea extract as an adjunct to non-surgical periodontal treatment. Odontology. 2016;104(1):89-97. doi:10.1007/s10266-014-0190-1. 
Rattanasuwan K, Rassameemasmaung S, Sangalungkarn V, Komoltri C. Clinical effect of locally delivered gel containing green tea extract as an adjunct to non-surgical periodontal treatment. Odontology. 2016;104(1):89-97. doi:10.1007/s10266-014-0190-1.

Duplicate / already

Ravi K, Divyashree P. Psidium guajava: A review on its potential as an adjunct in treating periodontal disease. Pharmacogn Rev. 2014;8(16):96-100. doi:10.4103/0973-7847.134233. included

Reading the tea leaves. University of California at Berkeley Wellness Letter. 2014;30(7):1-22p.

Recently published abstracts. Alternative Medicine Review. 2010;15(4):369-

Not addressing research question Not addressing research question $38012 p$

Not addressing research question

Rowan AD, Litherland GJ, Hui W, Milner JM. Metalloproteases as potential therapeutic targets in arthritis treatment. Expert Opin Ther Targets. 2008;12(1):1-18. doi:10.1517/14728222.12.1.1.

Not addressing research question

Sakanaka S, Okada Y. Inhibitory effects of green tea polyphenols on the production of a virulence factor of the periodontal-disease-causing anaerobic bacterium Porphyromonas gingivalis. J Agric Food Chem. 2004;52(6):16881692.

Not addressing research question

Sakanaka S, Okada Y. Inhibitory effects of green tea polyphenols on the production of a virulence factor of the periodontal-disease-causing anaerobic bacterium Porphyromonas gingivalis. J Agric Food Chem. 2004;52(6):16881692.

Sang QXA, Jin Y, Newcomer RG, et al. Matrix metalloproteinase inhibitors as prospective agents for the prevention and treatment of cardiovascular and neoplastic diseases. Curr Top Med Chem. 2006;6(4):289-316. doi:10.2174/156802606776287045.

Sarin S, Marya C, Nagpal R, Oberoi SS, Rekhi A. Preliminary Clinical Evidence of the Antiplaque, Antigingivitis Efficacy of a Mouthwash Containing $2 \%$ Green Tea - A Randomised Clinical Trial. Oral health prev. 2015;13(3):197-203. doi:10.3290/j.ohpd.a33447.

Schmuch J, Beckert S, Brandt S, et al. Extract from Rumex acetosa L. for prophylaxis of periodontitis: inhibition of bacterial in vitro adhesion and of gingipains of Porphyromonas gingivalis by epicatechin-3-O-(4beta->8)epicatechin-3-O-gallate (procyanidin-B2-Di-gallate). PLOS ONE. 2015;10(3):e0120130. doi:10.1371/journal.pone.0120130.

Not addressing Schmuch J, Beckert S, Brandt S, et al. Extract from Rumex acetosa L. for research question

Not addressing research question prophylaxis of periodontitis: inhibition of bacterial in vitro adhesion and of gingipains of Porphyromonas gingivalis by epicatechin-3-O-(4beta->8)epicatechin-3-O-gallate (procyanidin-B2-Di-gallate). PLOS ONE. 2015;10(3):e0120130. doi:10.1371/journal.pone.0120130.

Not addressing research question

Not addressing research question

Schmuch J, Beckert S, Brandt S, et al. Extract from Rumex acetosa L. for prophylaxis of periodontitis: Inhibition of bacterial in vitro adhesion and of gingipains of Porphyromonas gingivalis by epicatechin-3-O- $(4 \beta \rightarrow 8)$ epicatechin-3-O-gallate (procyanidin-B2-di-gallate). PLOS ONE. 2015;10(3). $\quad$ Not addressing doi:10.1371/journal.pone.0120130. research question

Schmuch J, Beckert S, Brandt S, Löhr G, Beikler T, Hensel A. Hydroalcoholic extracts from Rumex acetosa L. for prophylaxis of periodontitis: Inhibition of the bacterial adhesion and virulence factors of Porphyromonas gingivalis. $Z$ Phytother. 2016;37(4):151-159. doi:10.1055/s-0042-111129.

Not addressing research question

Shahzad M, Millhouse E, Culshaw S, Edwards CA, Ramage G, Combet E. Selected dietary (poly)phenols inhibit periodontal pathogen growth and biofilm formation. Food \& Function. 2015;6(3):719-729. doi:10.1039/c4fo01087f.

Not addressing research question

Shanbhag VK. Triphala in prevention of dental caries and as an antimicrobial in oral cavity- A review. Infect Disord Drug Targets. 2015;15(2):89-97.

Not addressing research question

Not addressing Shen CL, Yeh JK, Cao JJ, Chyu MC, Wang JS. Green tea and bone health: Evidence from laboratory studies. Pharmacol Res. 2011;64(2):155-161. doi:10.1016/j.phrs.2011.03.012.

Not addressing research question 
research question

Shuman IE. The gentle art of periodontal maintenance: A protocol using

Not addressing essential oils. Dent Today. 2003;22(4):50-57.

Signoretto C, Canepari P, Pruzzo C, Gazzani G. Woodhead Publ. Food Sci. Technol. Nutr. In: Wilson M, ed. Food Constituents and Oral Health: Current Status and Future Prospects. Cambridge: Woodhead Publ Ltd; 2009:240-262. doi:10.1533/9781845696290.2.240.

research question

Signoretto C, Canepari P, Stauder M, Vezzulli L, Pruzzo C. Functional foods and strategies contrasting bacterial adhesion. Current Opinion in Biotechnology. Not addressing 2012;23(2):160-167. doi:10.1016/j.copbio.2011.08.006. research question

Simran P, Rajkumar P. Nitric oxide and inflammatory periodontal disease. General Dentistry. 2015;63(2):34-407p.

Sirois M, Darby M, Tolle S. Understanding Muslim patients: cross-cultural dental hygiene care. Int J Dent Hygiene. 2013;11(2):105-11410p. doi:10.1111/j.16015037.2012.00559.x.

Snider J. Green tea may promote periodontal health. J Am Dent Assoc. 2009;140(7):838.

Not addressing research question

Not addressing research question

Not addressing research question Not addressing research question

Soory M. Inflammatory mechanisms and redox status in periodontal and cardiometabolic diseases: Effects of adjunctive nutritional antioxidants and statins. Infect Disord Drug Targets. 2012;12(4):301-315.

Spratt DA, Daglia M, Papetti A, et al. Evaluation of Plant and Fungal Extracts for Their Potential Antigingivitis and Anticaries Activity. J Biomed Biotechnol. 2012;2012:1-1212p.

Not addressing research question

Not addressing research question Not addressing research question Not addressing research question Not addressing research question

Studies on Periodontal Disease. New York: Springer Subapriya R, Nagini S. Medicinal properties of neem leaves: A review. Curr Med Chem Anti-Cancer Agents. 2005;5(2):149-156. doi:10.2174/1568011053174828.

Not addressing research question

Surathu N, Kurumathur AV. Traditional therapies in the management of periodontal disease in India and China. Periodontology 2000. 2011;56:14-24. doi:10.1111/j.1600-0757.2010.00369.x.

Not addressing research question

Tamura M, Saito H, Kikuchi K, et al. Antimicrobial Activity of Gel-Entrapped Catechins toward Oral Microorganisms. Biol Pharm Bull. 2011;34(5):638-643.

In vitro study

Tanaka K, Miyake Y, Sasaki S, et al. Beverage consumption and the prevalence of tooth loss in pregnant Japanese women: the Osaka Maternal and Child Health Study. Fukuoka Igaku Zasshi. 2008;99(4):80-89.

Not addressing research question

Tominari T, Matsumoto C, Watanabe K, et al. Epigallocatechin gallate (EGCG) suppresses lipopolysaccharide-induced inflammatory bone resorption, and protects against alveolar bone loss in mice. Febs Open Bio. 2015;5(1):522-527. doi:10.1016/j.fob.2015.06.003.

Not addressing research question

Tomofuji T, Ekuni D, Mizutani S, Morita M. Effects of Antioxidants on Periodontal Disease. (Ekuni D, Battino M, Tomofuji T, Putnins EE, eds.). New York: Springer; 2014:279-305. doi:10.1007/978-1-4614-9557-4_18.

Not addressing research question

Toyama T, Todoki K, Takahashi $\mathrm{Y}$, et al. Inhibitory effects of Jixueteng on $\mathrm{P}$. gingivalis-induced bone loss and osteoclast differentiation. Arch Oral Biol. 2012;57(11):1529-1536. doi:10.1016/j.archoralbio.2012.05.011.

Not addressing research question

Tsai HC, Li YC, Young TH, Chen MH. Citrus polyphenol for oral wound healing in oral ulcers and periodontal diseases. J Formos Med Assoc. 2016;115(2):100107. doi:10.1016/j.jfma.2015.01.003.

Turner L. 12 HEALTHY SHORTCUTS. Better Nutrition. 2012;74(3):38-447p.

Not addressing research question Not addressing research question 
Varela-Lopez A, Bullon P, Giampieri F, Quiles JL. Non-Nutrient, Naturally Occurring Phenolic Compounds with Antioxidant Activity for the Prevention and Treatment of Periodontal Diseases. Antioxidants. 2015;4(3):447-481. doi:10.3390/antiox4030447.

Varoni EM, Lodi G, Sardella A, Carrassi A, Iriti M. Plant Polyphenols and Oral Health: Old Phytochemicals for New Fields. Current Medicinal Chemistry. 2012;19(11):1706-1720

Not addressing research question

Not addressing research question

Venkateswara B, Sirisha K, Chava VK. Green tea extract for periodontal health. J Indian Soc Periodontol. 2011;15(1):18-22. doi:10.4103/0972-124X.82258.

Review

Venkateswara B, Sirisha K, Chava VK. Green tea extract for periodontal health. J Indian Soc Periodontol. 2011;15(1):18-22. doi:10.4103/0972-124X.82258.

Vervelle A, Mouhyi J, Del Corso M, Hippolyte M-P, Sammartino G, Ehrenfest DMD. Mouthwash solutions with microencapsuled natural extracts: Efficiency for dental plaque and gingivitis. Rev Stomatol Chir Maxillofac. 2010;111(3):148151. doi:10.1016/j.stomax.2009.09.014.

Viana GSB, Menezes SMS, Cordeiro LN, Matos FJA. Biological effects of pomegranate (Punica granatum L.), especially its antibacterial actions, against microorganisms present in the dental plaque and other infectious processes. In: Bioactive Foods in Promoting Health. Elsevier Inc.; 2010:457-478. doi:10.1016/B978-0-12-374628-3.00031-1.

Review/Duplicate

Vyas A, Syeda K, Ahmad A, Padhye S, Sarkar FH. Perspectives on medicinal properties of mangiferin. Mini-Rev Med Chem. 2012;12(5):412-425. Walker WA, Martens EC, Sherman PM, Lampe JW, Hullar MAJ, Wu CD. Functional foods for health promotion: microbes and health: extended abstracts from the 11th Annual Conference on Functional Foods for Health Promotion, April 2008. Nutrition Reviews. 2009;67(1):40-489p.

Not addressing research question

Wang SP, Zhang JM, Chen MW, Wang YT. Delivering flavonoids into solid tumors using nanotechnologies. Expert Opinion on Drug Delivery. 2013;10(10):1411-1428. doi:10.1517/17425247.2013.807795.

Not addressing research question

Not addressing research question

Wen WC, Kuo PJ, Chiang CY, Chin YT, Fu MMJ, Fu E. Epigallocatechin-3Gallate Attenuates Porphyromonas gingivalis Lipopolysaccharide-Enhanced Matrix Metalloproteinase-1 Production Through Inhibition of Interleukin-6 in Gingival Fibroblasts. Journal of Periodontology. 2014;85(6):868-875. doi:10.1902/jop.2013.120714.

Not addressing research question

Whelton H. Woodhead Publ. Food Sci. Technol. Nutr. In: Wilson M, ed. Food Constituents and Oral Health: Current Status and Future Prospects. Cambridge: Woodhead Publ Ltd; 2009:488-528. doi:10.1533/9781845696290.3.488.

Not addressing research question

Not addressing research question

Wojtaszek C. Management of chemotherapy-induced stomatitis. Clinical Journal of Oncology Nursing. 2000;4(6):263-28210p.

Wolle CFB, Zollmann LD, Etges A, Vitalis GS, Leite CE, Campos MM. Effects of the Antioxidant Agent Tempol on Periapical Lesions in Rats with Doxorubicininduced Cardiomyopathy. J Endod. 2012;38(2):191-195. doi:10.1016/j.joen.2011.11.007.

Wood N. Oral health -- how to reduce risks of periodontitis. Positive Health. 2006;(127):30-356p.

Not addressing research question

Not addressing research question

Wyganowska-ŚWjAtkowska M, Surdacka A, Skrzypczak-Jankun E, Jankun J. The plasminogen activation system in periodontal tissue (Review). Int J Mol Med. 2014;33(4):763-768. doi:10.3892/ijmm.2014.1653.

Xu X, Zhou XD, Wu CD. Tea Catechin EGCg Suppresses the mgl Gene Associated with Halitosis. Journal of Dental Research. 2010;89(11):1304-1308. doi:10.1177/0022034510378682.

Yamanaka A, Kouchi T, Kasai K, Kato T, Ishihara K, Okuda K. Inhibitory effect of cranberry polyphenol on biofilm formation and cysteine proteases of Porphyromonas gingivalis. J Periodontal Res. 2007;42(6):589-592. doi:10.1111/j.1600-0765.2007.00982.x.

Yiannakopoulou EC. Recent patents on antibacterial, antifungal and antiviral properties of tea. Recent Pat Anti-Infect Drug Discov. 2012;7(1):60-65. doi:10.2174/157489112799829738.

Not addressing research question

Not addressing research question

Not addressing research question

Not addressing research question

Not addressing research question

Not addressing research question 
Yoshinaga Y, Ukai T, Nakatsu S, et al. Green tea extract inhibits the onset of periodontal destruction in rat experimental periodontitis. J Periodontal Res. 2014;49(5):652-6598p. doi:10.1111/jre.12147.

You SQ. [Study on feasibility of Chinese green tea polyphenols (CTP) for preventing dental caries]. Chung Hua Kou Chiang Hsueh Tsa Chih.

In vitro study 1993;28(4):197-9-254.

Yuan FL, Xu RS, Jiang DL, et al. Leonurine hydrochloride inhibits osteoclastogenesis and prevents osteoporosis associated with estrogen deficiency by inhibiting the NF-KB and PI3K/Akt signaling pathways. Bone. 2015;75:128-137. doi:10.1016/j.bone.2015.02.017.

Yun JH, Kim CS, Cho KS, Chai JK, Kim CK, Choi SH. (-)-Epigallocatechin gallate induces apoptosis, via caspase activation, in osteoclasts differentiated from RAW 264.7 cells. J Periodontal Res. 2007;42(3):212-218.

doi:10.1111/j.1600-0765.2006.00935.x.

Not addressing research question

Yun JH, Pang EK, Kim CS, et al. Inhibitory effects of green tea polyphenol (-)epigallocatechin gallate on the expression of matrix metalloproteinase- 9 and on the formation of osteoclasts. J Periodontal Res. 2004;39(5):300-307. doi:10.1111/j.1600-0765.2004.00743.x.

Not addressing research question

Not addressing research question

Yuvaraja M, Reddy NR, Kumar PM, Ravi KS, Alqahtani N. Thermoreversible gel for intrapocket delivery of green tea catechin as a local drug delivery system: An original research. J. 2016;7(4):139-143.

full text assessment

Zagorouiko V, Mizin V, Bogadelnikov I, Ogay U. The Dietary Grape Polyphenol

Concentrate "ENOANT" Enables Protection Against Biological Agents. In:

Dishovsky C, Pivovarov A, eds. Counteraction to Chemical and Biological

Terrorism in East European Countries. Dordrecht: Springer; 2009:167-176. doi:10.1007/978-90-481-2342-1_21.

Not addressing

research question

Zhao L, La VD, Grenier D. Antibacterial, antiadherence, antiprotease, and antiinflammatory activities of various tea extracts: potential benefits for periodontal diseases. J med food. 2013;16(5):428-436. doi:10.1089/jmf.2012.0207.

Not addressing research question

Not addressing research question 


\begin{tabular}{|c|c|c|c|c|c|c|c|c|}
\hline Phase of the study & Item & Question & Answers & $\begin{array}{l}\text { Funosas } \\
\text { ER } \\
(2005)\end{array}$ & $\begin{array}{l}\text { Kudva } \\
\text { (2011) }\end{array}$ & $\begin{array}{l}\text { Chava } \\
\text { VK } \\
(2013)\end{array}$ & $\begin{array}{l}\text { Hattarki } \\
\text { SA } \\
\text { (2013) }\end{array}$ & $\begin{array}{l}\text { Rattanas } \\
\text { uwan K } \\
(2014)\end{array}$ \\
\hline \multirow[t]{2}{*}{ Registration } & Ethic committee & Done? & $0=$ no/NR; $1=$ yes & 0 & 0 & 1 & 1 & 1 \\
\hline & Informed consent & Done? & $0=$ no/NR; $1=$ yes & 1 & 1 & 1 & 1 & 1 \\
\hline Funding & Independent funding & Described? & $\begin{array}{l}0=\text { private/industry/NR; } \\
1=\text { university/government/self }\end{array}$ & 1 & 1 & 1 & 1 & 1 \\
\hline \multirow[t]{3}{*}{ Randomization } & Sequence generation & Done? & $0=$ inadequate $/ \mathrm{NR} ; 1=$ adequate & 0 & 0 & 1 & 0 & 0 \\
\hline & Allocation concealment & Done? & $0=$ inadequate $/ \mathrm{NR} ; 1=$ adequate & & & & & \\
\hline & & & & 0 & 0 & 1 & 0 & 0 \\
\hline \multirow[t]{4}{*}{ Blinding } & Patient & Done? & $0=$ inadequate $/ \mathrm{NR} ; 1=$ adequate & 0 & 0 & 0 & 0 & 0 \\
\hline & Examiner & Done? & $0=$ inadequate $/ \mathrm{NR} ; 1=$ adequate & & & & & \\
\hline & Therapist & Done? & $0=$ inadequate $/ \mathrm{NR} ; 1=$ adequate & 0 & 0 & 0 & 0 & 1 \\
\hline & & & & 0 & 0 & 0 & 0 & 1 \\
\hline Participants & $\begin{array}{l}\text { Clear definition of eligibility } \\
\text { criteria }\end{array}$ & Described? & $0=$ no; $1=$ yes & 1 & 1 & 1 & 1 & 1 \\
\hline Statistics & Appropriate statistical analysis & Done? & $0=$ no/NR; $1=$ yes & 1 & 1 & 1 & 1 & 1 \\
\hline Calibration & Calibration & Done? & $0=$ no/NR; $1=$ yes & 1 & 0 & 1 & 0 & 1 \\
\hline \multirow[t]{3}{*}{$\begin{array}{l}\text { Completeness of } \\
\text { outcome data }\end{array}$} & $\begin{array}{l}\text { Sample size calculation \& } \\
\text { power analysis }\end{array}$ & Done? & $0=$ no/NR; $1=$ yes & 0 & 0 & 1 & 0 & 1 \\
\hline & $\begin{array}{l}\text { Missing outcome data } \\
\text { reported }\end{array}$ & Reported? & $0=$ no; $1=$ yes $/$ no dropouts & 1 & 1 & 1 & 1 & 1 \\
\hline & $\begin{array}{l}\text { Reasons for dropouts } \\
\text { specified }\end{array}$ & Reported? & $0=$ no; $1=$ yes & 0 & 0 & 1 & 0 & 1 \\
\hline \multirow[t]{10}{*}{ Other sources of bias } & $\begin{array}{l}\text { Were systemic diseases and } \\
\text { medication reported? } \\
\text { Were clinical periodontal }\end{array}$ & Reported? & $0=$ no; $1=$ yes & 0 & 0 & 0 & 0 & 1 \\
\hline & conditions specified? & Reported? & $0=$ no; $1=$ yes & 1 & 1 & 1 & 1 & 1 \\
\hline & Was smoking reported? & Reported? & $0=$ no; $1=$ yes & 0 & 1 & 1 & 1 & 1 \\
\hline & Were oral hygiene levels & & & & & & & \\
\hline & reported? & Reported? & $0=$ no; $1=$ yes & 1 & 1 & 1 & 1 & 1 \\
\hline & Were tooth types specified? & Reported? & $0=$ no; $1=$ yes & 0 & 0 & 0 & 0 & 0 \\
\hline & Were sources of green tee & & & & & & & \\
\hline & agents reported? & Reported? & $0=$ no; $1=$ yes & 1 & 1 & 1 & 1 & 1 \\
\hline & $\begin{array}{l}\text { Was percentage and } \\
\text { frequency of agent application }\end{array}$ & & & & & & & \\
\hline & reported? & Reported? & $0=$ no; $1=$ yes & 1 & 1 & 1 & 1 & 1 \\
\hline
\end{tabular}

a, adapted from Graziani et al. 2012; nr, not reported. 


\section{Appendix}

\section{PRISMA Checklist}

\begin{tabular}{|c|c|c|c|}
\hline Section/topic & $\overline{\#}$ & Checklist item & $\begin{array}{l}\text { Reported } \\
\text { on page \# }\end{array}$ \\
\hline \multicolumn{4}{|l|}{ TITLE } \\
\hline Title & 1 & $\begin{array}{l}\text { Identify the report as a systematic review, meta-analysis, or } \\
\text { both. }\end{array}$ & 1 \\
\hline \multicolumn{4}{|l|}{ ABSTRACT } \\
\hline $\begin{array}{l}\text { Structured } \\
\text { summary }\end{array}$ & 2 & $\begin{array}{l}\text { Provide a structured summary including, as applicable: } \\
\text { background; objectives; data sources; study eligibility criteria, } \\
\text { participants, and interventions; study appraisal and synthesis } \\
\text { methods; results; limitations; conclusions and } \\
\text { implications of key findings; systematic review registration } \\
\text { number. }\end{array}$ & 2 \\
\hline \multicolumn{4}{|l|}{ INTRODUCTION } \\
\hline Rationale & 3 & $\begin{array}{l}\text { Describe the rationale for the review in the context of what is } \\
\text { already known. }\end{array}$ & 4,5 \\
\hline Objectives & 4 & $\begin{array}{l}\text { Provide an explicit statement of questions being addressed } \\
\text { with reference to participants, interventions, comparisons, } \\
\text { outcomes, and study design (PICOS). }\end{array}$ & 6 \\
\hline \multicolumn{4}{|l|}{ METHODS } \\
\hline $\begin{array}{l}\text { Protocol and } \\
\text { registration }\end{array}$ & 5 & $\begin{array}{l}\text { Indicate if a review protocol exists, if and where it can be } \\
\text { accessed (e.g., Web address), and, if available, provide } \\
\text { registration information including registration number. }\end{array}$ & 5 \\
\hline Eligibility criteria & 6 & $\begin{array}{l}\text { Specify study characteristics (e.g., PICOS, length of follow-up) } \\
\text { and report characteristics (e.g., years considered, language, } \\
\text { publication status) used as criteria for eligibility, giving } \\
\text { rationale. }\end{array}$ & 6 \\
\hline $\begin{array}{l}\text { Information } \\
\text { sources }\end{array}$ & 7 & $\begin{array}{l}\text { Describe all information sources (e.g., databases with dates of } \\
\text { coverage, contact with study authors to identify additional } \\
\text { studies) in the search and date last searched. }\end{array}$ & 6 \\
\hline Search & 8 & $\begin{array}{l}\text { Present full electronic search strategy for at least one } \\
\text { database, including any limits used, such that it could be } \\
\text { repeated. }\end{array}$ & 6 \\
\hline Study selection & 9 & $\begin{array}{l}\text { State the process for selecting studies (i.e., screening, } \\
\text { eligibility, included in systematic review, and, if applicable, } \\
\text { included in the meta-analysis). }\end{array}$ & 6,7 \\
\hline $\begin{array}{l}\text { Data collection } \\
\text { process }\end{array}$ & 10 & $\begin{array}{l}\text { Describe method of data extraction from reports (e.g., piloted } \\
\text { forms, independently, in duplicate) and any processes } \\
\text { for obtaining and confirming data from investigators. }\end{array}$ & 7 \\
\hline Data items & 11 & $\begin{array}{l}\text { List and define all variables for which data were sought (e.g., } \\
\text { PICOS, funding sources) and any assumptions and } \\
\text { simplifications made. }\end{array}$ & 7,8 \\
\hline $\begin{array}{l}\text { Risk of bias in } \\
\text { individual studies }\end{array}$ & 12 & $\begin{array}{l}\text { Describe methods used for assessing risk of bias of individual } \\
\text { studies (including specification of whether this was } \\
\text { done at the study or outcome level), and how this information } \\
\text { is to be used in any data synthesis. }\end{array}$ & 7 \\
\hline $\begin{array}{l}\text { Summary } \\
\text { measures }\end{array}$ & 13 & $\begin{array}{l}\text { State the principal summary measures (e.g., risk ratio, } \\
\text { difference in means). }\end{array}$ & 7 \\
\hline $\begin{array}{l}\text { Synthesis of } \\
\text { results }\end{array}$ & 14 & $\begin{array}{l}\text { describe the methods of handling data and combining results } \\
\text { of studies, if done, including measures of consistency }\left(e . g ., I^{2}\right)\end{array}$ & 7,8 \\
\hline $\begin{array}{l}\text { Risk of bias } \\
\text { across studies }\end{array}$ & 15 & $\begin{array}{l}\text { for each meta-analysis. } \\
\text { Specify any assessment of risk of bias that may affect the } \\
\text { cumulative evidence (e.g., publication bias, selective }\end{array}$ & 7 \\
\hline
\end{tabular}


reporting within studies).

Additional

Describe methods of additional analyses (e.g., sensitivity or

7,8 analyses subgroup analyses, meta-regression), if done, indicating which were pre-specified.

\section{RESULTS}

Study selection

17 Give numbers of studies screened, assessed for eligibility, and included in the review, with reasons for exclusions at

Study characteristics

Risk of bias within studies Results of individual studies

Synthesis of results

Risk of bias across studies Additional analysis

\section{DISCUSSION}

Summary of evidence each stage, ideally with a flow diagram.

18 For each study, present characteristics for which data were extracted (e.g., study size, PICOS, follow-up period) and provide the citations.

19 Present data on risk of bias of each study and, if available, any outcome level assessment (see item 12).

20 For all outcomes considered (benefits or harms), present, for each study: (a) simple summary data for each intervention group (b) effect estimates and confidence intervals, ideally with a forest plot.

21 Present results of each meta-analysis done, including confidence intervals and measures of consistency.

22 Present results of any assessment of risk of bias across studies (see Item 15).

23 Give results of additional analyses, if done (e.g., sensitivity or subgroup analyses, meta-regression [see Item 16]).
Limitations

Conclusions

\section{FUNDING}

Funding
Summarize the main findings including the strength of evidence for each main outcome; consider their relevance to key groups (e.g., healthcare providers, users, and policy makers)

25 Discuss limitations at study and outcome level (e.g., risk of bias), and at review-level (e.g., incomplete retrieval of identified research, reporting bias).

26 Provide a general interpretation of the results int he context of 12 other evidence, and implications for future research.

27 Describe sources of funding for the systematic review and other support (e.g., supply of data); role of funders for the systematic review. 